Association of Arab Universities Journal of Engineering Sciences

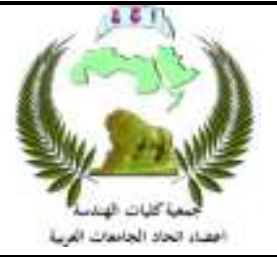

التنقيب عن العناصر المتكررة بالاعتماد على تطوير خوارزمية FP-growth و استخدام تقنية MapReduce

\author{
زكريا مهروسة2، ديما مفتي الثوافعة2، حسن قزاز3* \\ قسم هندسة الحو اسيب، كلية الهندسة الكهربائية و الالكترونية، جامعة حلب، سوريا، Rmahrousa@hotmail.com. \\ قدم هندسة الحو اسيب، كلية الهندسة الكهربائية و الالكترونية، جامعة حلب، سوريا، gmail.com.dima.mufti@ \\ قسم هندسة الحو اسيب، كلبة الهندسة الكهربائبة والالكترونية، جامعة حلب، سوريا، gmail.com.kazzazhasan@ قو
}

kazzazhasan@gmail.com الباحث الممثل: حسن قزاز *

نشر في: 31 اذار 2021

الخلاصة - إيجاد قواعد الترابط (Association Rules) في البيانات الضخمة (Big Data) عملية مهمة في مجال التنقيب عن البيانات (Data Mining) و اكتشاف المعرفة (Knowledge Discovery). تنتج البيانات الضخمة من النمو اليومي المضطرد (الأسي) للبيانات،

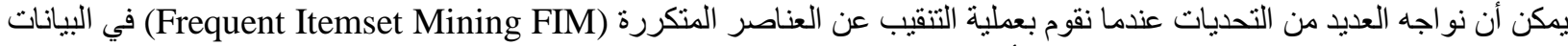

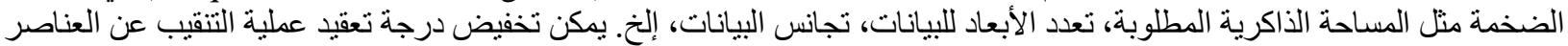

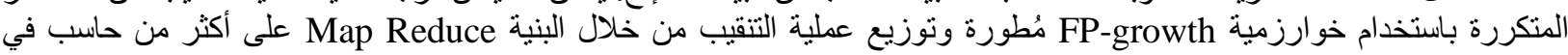

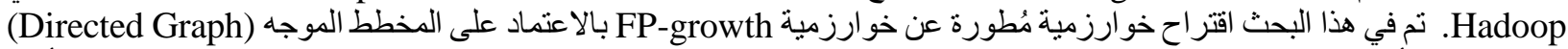

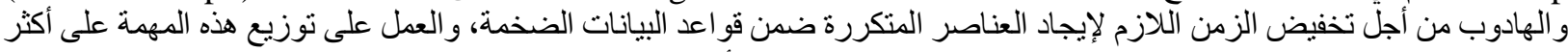

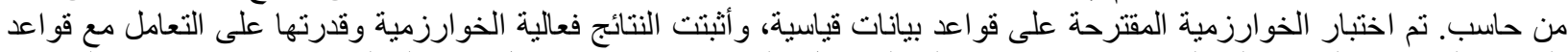

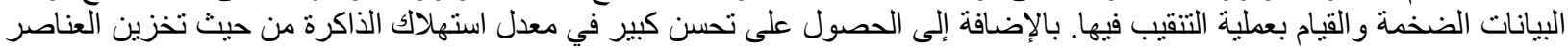
المتكررة، بالإضافة إلى التعقيد بالنسبة إلى الزمن.

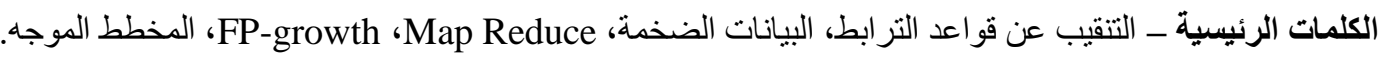

المنكررة و إيجاد قو اعد الترابط، خصوصاً عند التعامل مع قواعد البيانات الضخمة [4][6]ة فايجاد.

حيث يمكن التغلب على مشاكل التنقيب التسلسلي عن العناصر المتكررة من التناصن خلال الدمج بين مفهوم الحوسبة السحابية والتنقيب عن العناصر النيب العنكررة.

\section{2.}

إن إيجاد العناصر المتكررة مرحلة مهمة في عملية التنقيب عن قو اعد الترابط

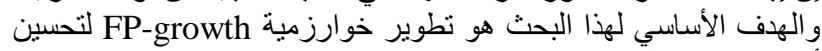

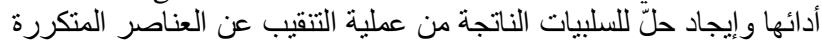

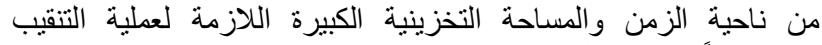

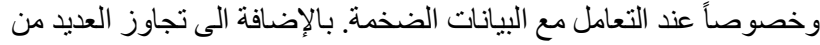

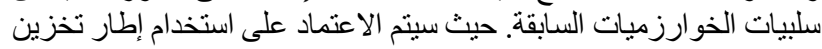

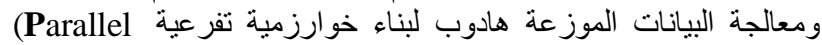
(Graph Frequent Pattern PGFP-growth)

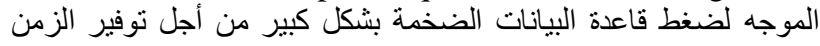

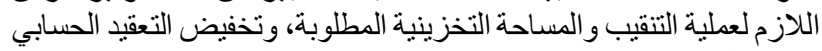

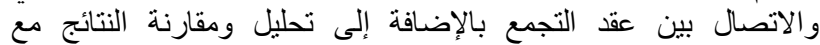
الخو ارزميات السابقة.

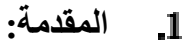

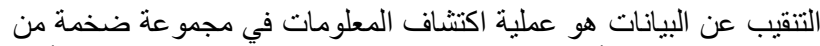

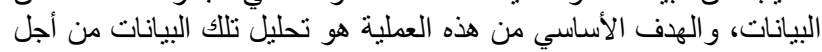

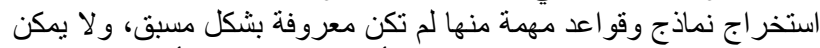

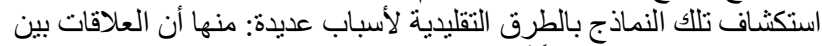

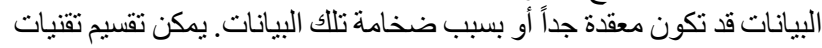

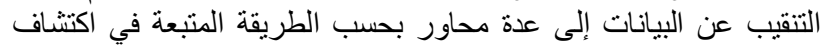
المعرفة. حيث يوجد تقنيات التنقيب عن قواعد الترابط التئ Association) Rules Mining ARM) وClustering)

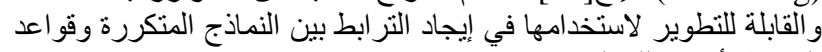
الترابط، أشهر تللك الخوارزميات هي خوارزمية الأنية (Apriori Algorithm)

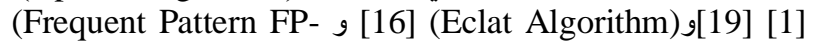
[7 growth)

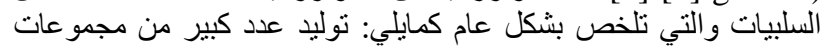

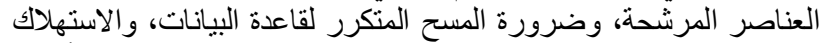

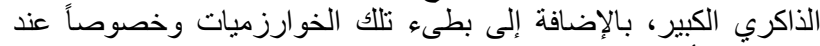
التعامل مع أحجام بيانات ضخمة مما يؤدي إلى فثل عملية التئ التنقيب.

لذلك تم العمل على إيجاد استراتيجيات لمعالجة المشاكل السابقة ومنها

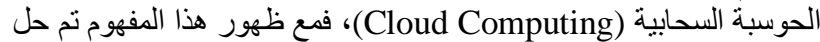

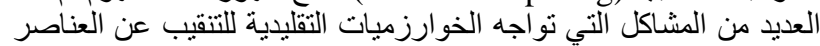


الخوارزميات المعتمدة على القيام بعبور وحيد لقاعدة البيانات: $\bullet$

خوارزمية العبور الوحبد لشجرة CP-Tree، قدمت مثال عن بنية شجرية

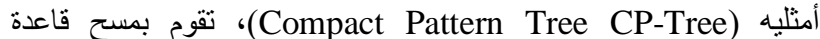
المعطيات من خلال طور الإدخال (Insertion phase) وتطبق نفس الآلية

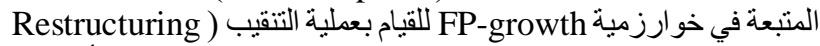

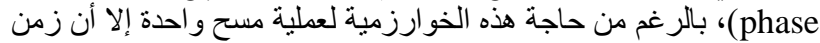
عملية إنشاء Header Table وبناء الثجرة بزداء منداد مع زيادة حجم قاعدة المعطيات بشكل أسي][21]

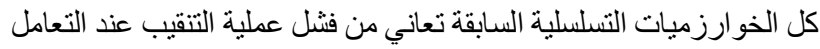

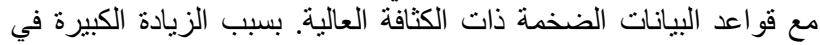
المساحة الذاكرية اللازمة لعملية المعالجة.

\section{الخوارزميات الناصة بالبيانات الضخمة:}

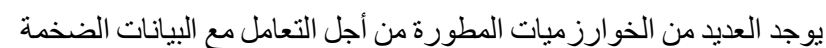

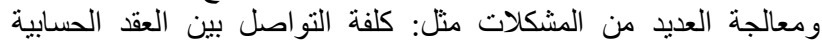
(Communication cost)

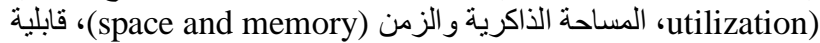

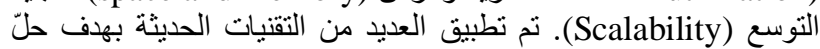

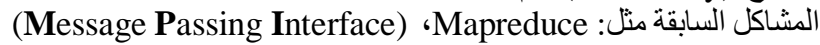
(Field-Programmable Gate Array) FPGA) ،MPI الرسومياتGraphics Processing Unit GPU) ، استخدام العناقيد

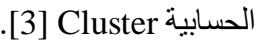

\section{الخوارزميات المعتمدة على شريحة FPGAs:}

تم العمل على تطوير خوارزمية FP-growth تفر عية من خلال توزيع مهمة التنقيب عن العناصر المتكررة عبر شريحة شئرة مصفوفة البو ابات القابلة للبرمجة

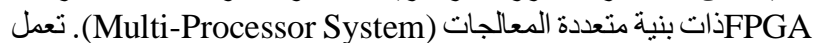
الخوارزمية على تقسيم الشريحة إلى عدة وحدات معات معالجة (Processing) Entities (PEs))

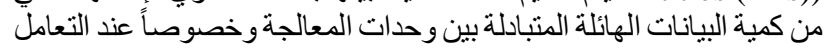

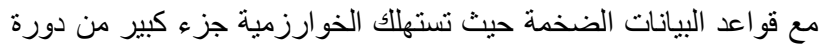
الساعة من أجل تبادل البيانات بين وحدات المعالجة [22].

\section{الخوارزميات المعتمدة على وحدة معالجة الرسومياتGPUs :}

عملت العديد من الأبحاث على استخدام وحدة معالجة الرسوميات نظراً

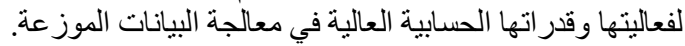

خو ارزمية Jiang H and Meng H.) Parallel FP-growth، تعتمد

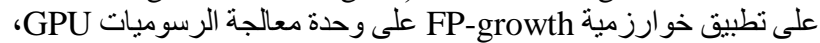
والتي تتألف من آلاف الأنوية الحسابية (Computing Cores) القابلة ولنة

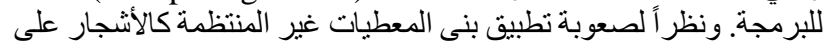

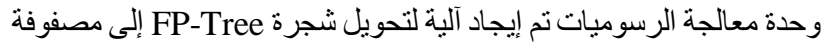
ثنائية الأبعاد FP-array بشكل الرسوديات منطقي [10].

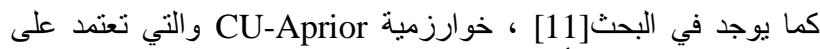

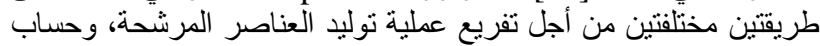

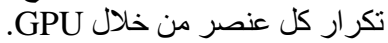

النسخ الموزعة من خوارزمية Aprior و

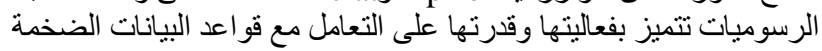

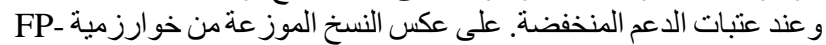
growth

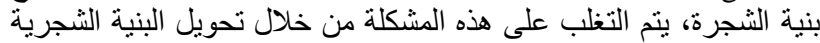

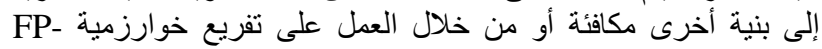

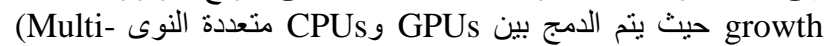

مفهوم إيجاد العناصر المتكررة:

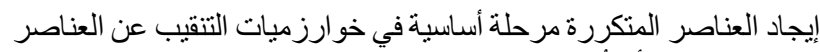

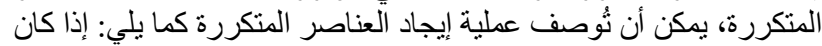

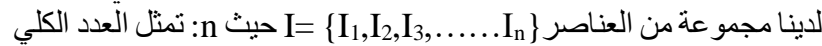

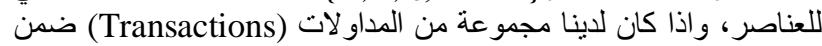
قاعدة البيانات

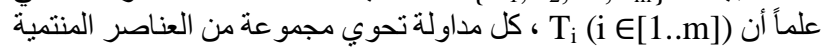

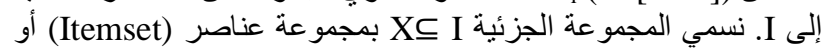

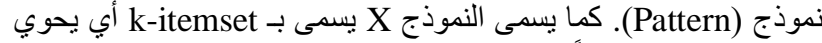

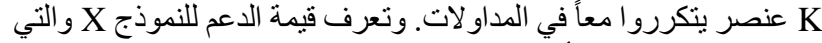

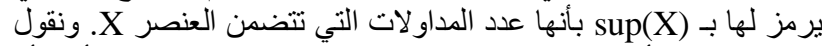

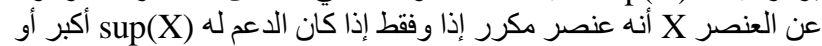

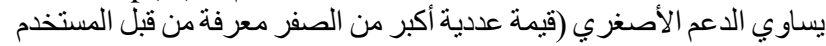

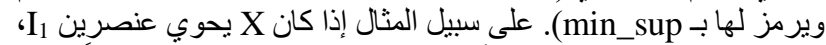

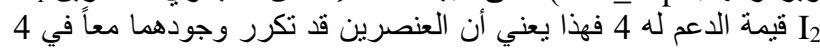
مداو لات. أما قو اعد التر ابط فتأخذ الثكل

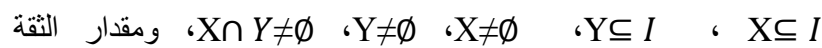
لأي فاعدة هو (Confidence)

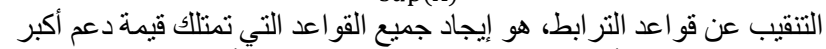

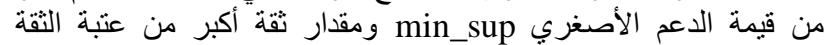
الأصغرية أيضاً][7] [8] [16 [16 ]

$$
\text { الدر اسة المرجعية والأبحاث السابقة: }
$$

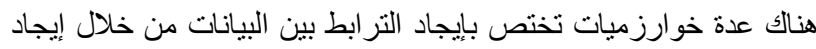

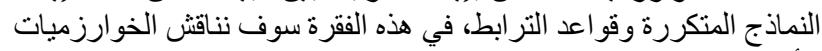

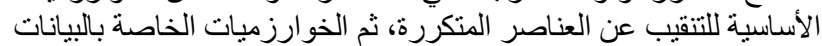
الضخمة.

$$
\text { الخوازميات الأساسية للتنقيب عن البيانات: }
$$

يمكن تقسيم الخوارزميات الأساسية للتنقيب عن العناصر المتكررة حسب إلى التبات

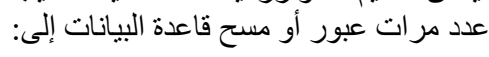

$$
\text { الخوارزميات المعتمدة على العبور المتكرر لقاعدة البيانات: }
$$

خوارزمية Apriori و التي تستخدم طريقة التوليد والاختبار and Test

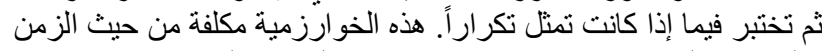

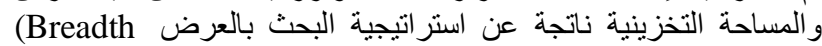
First Search)

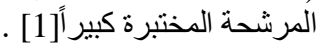

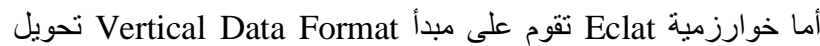

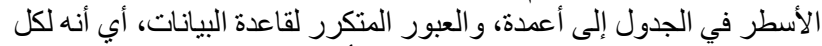

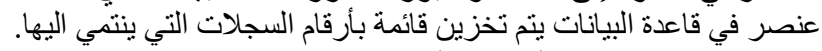

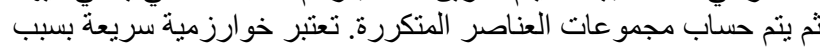

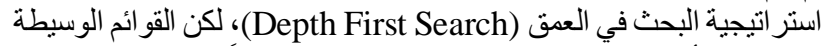

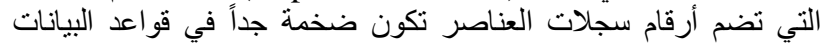
الضخمة] الضئ

\section{الخوارزميات المعتمدة على القيام بعبورين لقاعدة البيانات:}

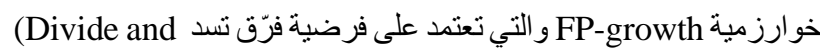

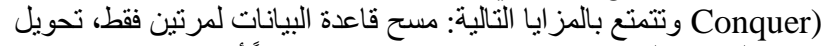

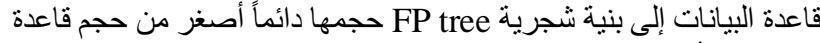

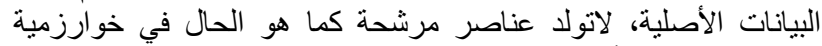
Apriori ، وتعتبر أسر ع من الخوارزميات السابقة] [7 [8] [14]. 


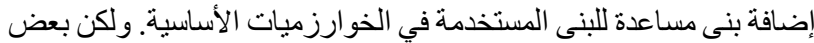

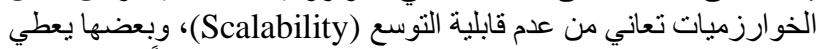

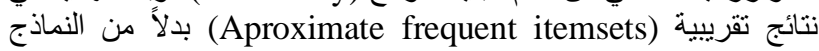

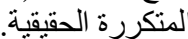

سوف يتناول بحثنا تطوير خوارزمية FP-growth من خوارزميات التنقيب

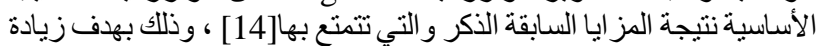

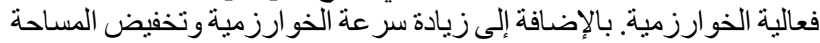

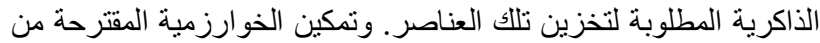

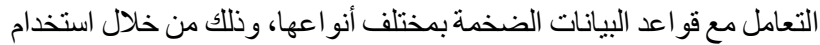

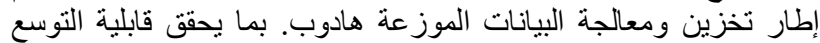
وسر عة تفوق التطبيق التقليدي لخو ارزمية (Scalable) حاسب واحد فقط والعمل على موازنة الأحمال بين العقد الحسابية.

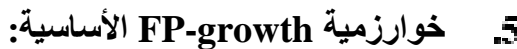

تعمل خوارزمية FP-growth الأساسية وفق الخطوات التالية:

المرور الأول لقاعدة البيانات لإيجاد 1-itemsets (مجموعة تتضمن

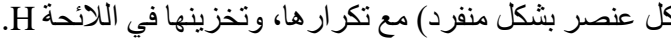

الحصول على العناصر المتكررة والتي يكون تكرارها أكبر من عنبة

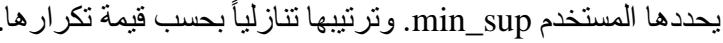

3. ترنيب عناصركل مداولة في قاعدة البيانات وفق اللائحة H.

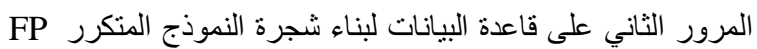
tree

إنشاء جذر الشجرة وتسميته null.

لكل سجل من سجلات قاعدة البيانات، يتم ترتيب العناصر المتكررة فيه بحسب الترتيب في اللائحة

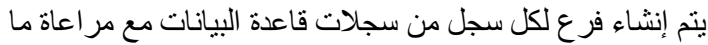

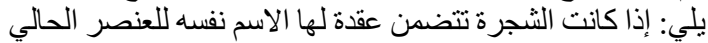

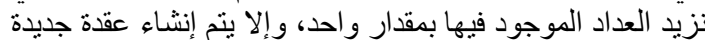
يكون التكر ار فيها مساوياً الو احد مع ربطها مع والح العقدة الأب لها.

التنقيب في شجرة FP tree: لكل نموذج بطول 1 (نموذج لاحق ابتدائي Conditional (prefix pattern

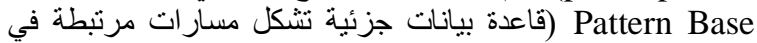

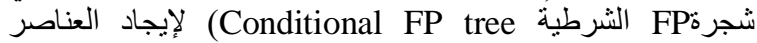

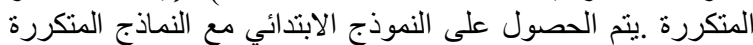

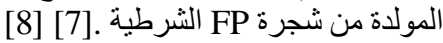

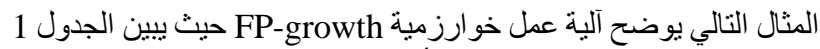

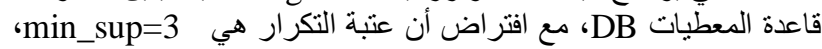

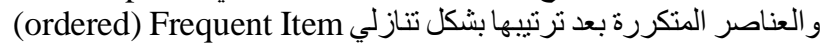

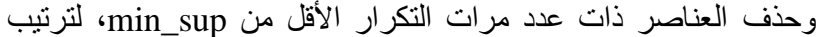

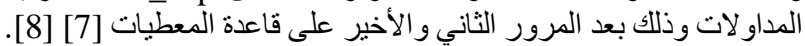

الجدول 1: مداو لات قاعدة المعطيات DB.

\begin{tabular}{|c|c|c|}
\hline TID & Transactions & $\begin{array}{c}\text { (ordered) Frequent } \\
\text { Item }\end{array}$ \\
\hline T100 & f, a, c, d, g, i, m, p & f, c, a, m, p \\
\hline T200 & a, b, c, f, l, m, o & f, c, a, b, m \\
\hline T300 & b, f, h, j, o & f, b \\
\hline
\end{tabular}

\section{الخوارزميات المعتمدة على العناقيا الحسابية CPUs:}

من أوائل الخوارزميات في هذا المجال، الخوارزمية المقترحة في المرجع

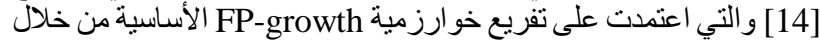

البنية MapReduce.

الخو ارزمية المقدمة (Alhamodi A. et al.) هي عبارة

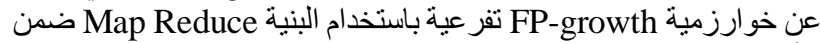

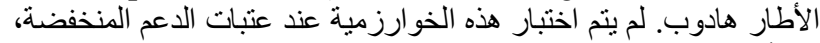

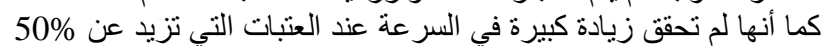

خوارزمية S-FPG (Spark FPGrowth) للتنقيب عن النماذج المتكررة

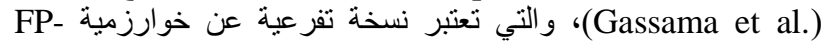

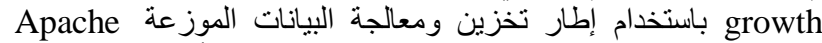
Spark TM

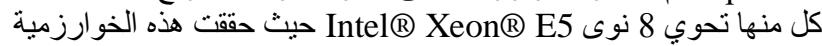
زيادة في السرعة بمقدار 6 أضعاف عن خوارزمية FP-growth تفره

باستخدام البنية في في عap Reduce.

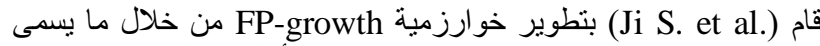
بالمسار ات المشتركة (Sharing Path) وذللك من أجل التقليل من مسح قاعدة

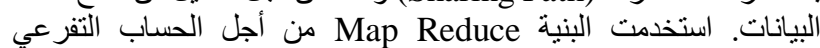

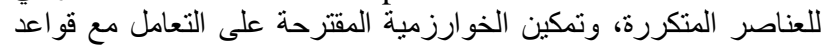

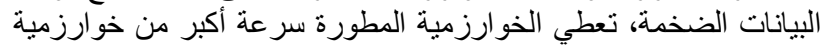
FP-growth

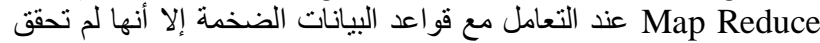

زيادة ملحوظة في السرعة مع قو اعد البيانات المتوسطة و الصغيرة [9] الصان.

قدم (Kulkarni P G et al.) خوارزمية تفرعية معدلة عن خوارزمية

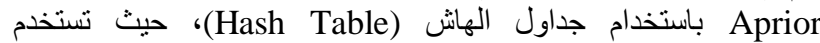

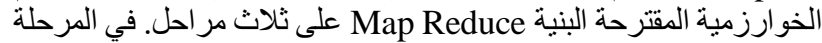

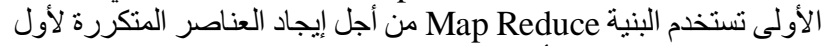

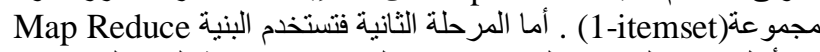

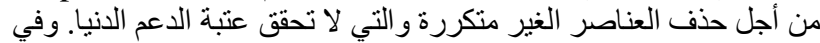

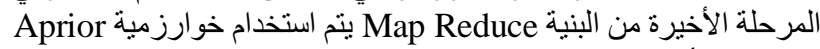

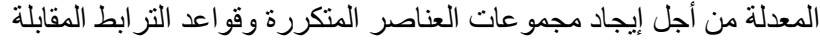

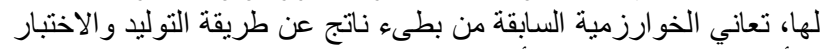

المأخوذة من الخوارزمية الأساسية [12].

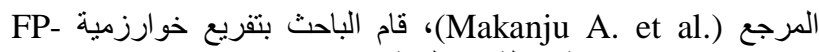

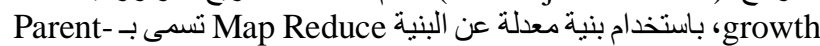
Child MapReduce

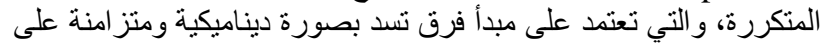

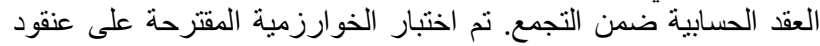

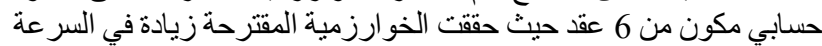
تقدر بثلاثة أضعاف سر عة خوارزمية FP-growth تفر عية باستخدام البنية عip Reduce

قام (Nikam P. V. et al. بتقديم خو ارزمية للتنقيب عن العناصر المنكررة

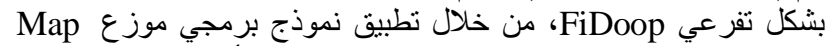
Reduce

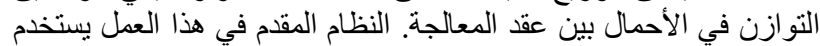

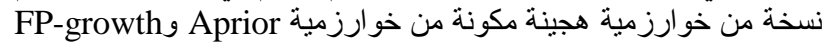

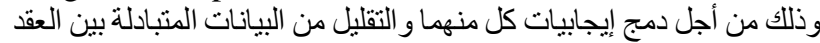

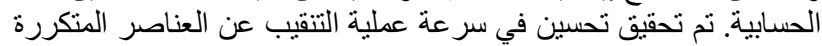

في معظم الخوارزميات السابقة تم التغلب على مشكلة المساحة الذاكرية

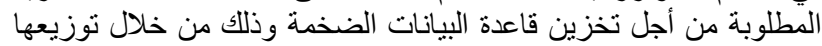

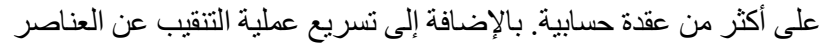
المتكررة، على الرغم من زيادة تعقيد بعض الخو الارزميات السية السابقة من خلال 


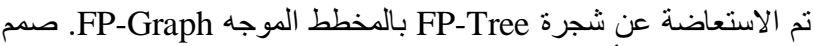
FP-Graph

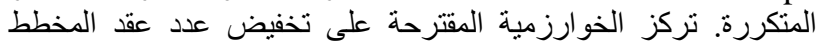

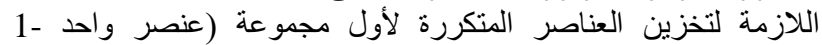
(itemsets

يمكن أن نُعرف المخطط (FP-Graph): بأنه عبارة عن مخطط موجه Ge ليحوي مجموعة من العقد (Eertices)، ومجمو عة من الحو اف المرتبة (Edge)

و إذا كان لدينا قاعدة البيانات برمز لها بـ

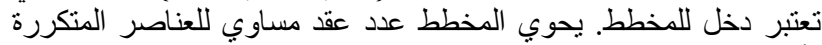

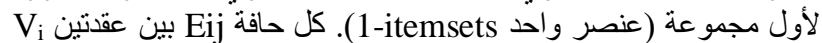

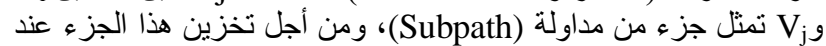

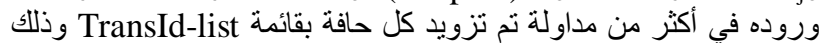

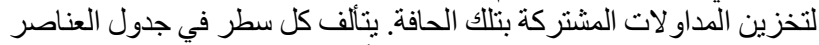

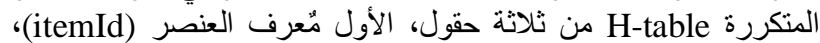

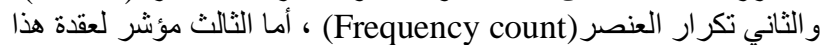
العنصر في المخطط(itemNodePointer).

كل عقدة في المخطط تتألف من حقلين، الأول مُعرف العنصر ، و الثاني عبارة

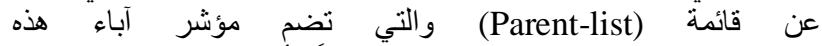
العقدة(ParentNodePointer). ذكرنا سابقاً بأن الخوارزمية المقترحة

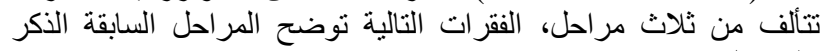

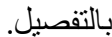

\section{I}

بناء المخطط FP-Graph من قاعدة البيانات ينطلب نرتيب العناصر

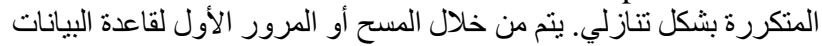

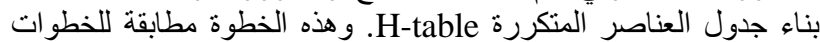
الأولى في خواه ارزمية FP-growth الأساسية وبدون أي تعديل.

\section{بناء المخطط FP-Graph:}

6.2

يتم بناء المخطط الخاص بالعناصر المتكررة دون الحاجة إلى أي بنية مساعدة

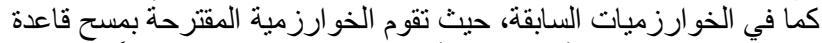

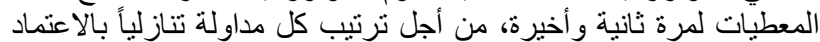

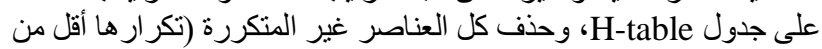

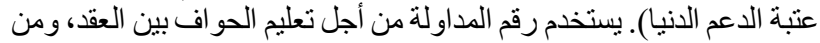

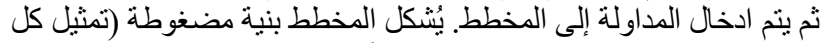

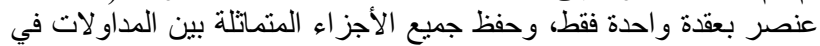

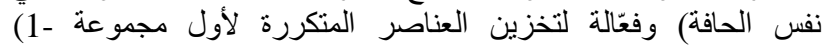

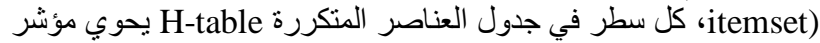

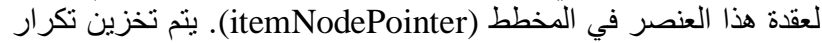

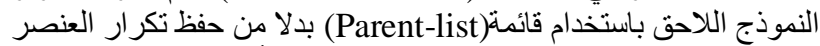

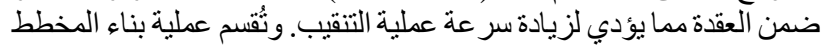

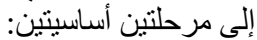

1. تهيئة العقد وربطها مع المؤشر itemNodePointer: الخطوات من 1 إلى 2.3 .4 في الثيفرة الز ائفة الموضحة لاحقاً.

حفظ المداولات في المخطط: الخطوات من 3 إلى 3.3 في الثيفرة

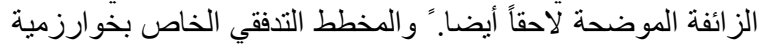
بناء المخطط موضح في الثكل 3.

بما أن المخطط لا يحوي عقدة جذر (Root) يتم العبور عبر العقد من خلال جدول H-table. أما البحث عن النموذج اللاحق (Rrefix Pattern) ينطلب الئب

\begin{tabular}{|c|c|c|}
\hline T400 & b, c, k, s, p & c, b, p \\
\hline T500 & a, f, c, e, l, p, m, n & f, c, a, m, p \\
\hline
\end{tabular}

أما الثكل 1 يبين جدول Header-table و الذي يحوي العناصر المتكررة، وشجرة FP-Tree التي تمثل قاعدة المعطيات السابقة.

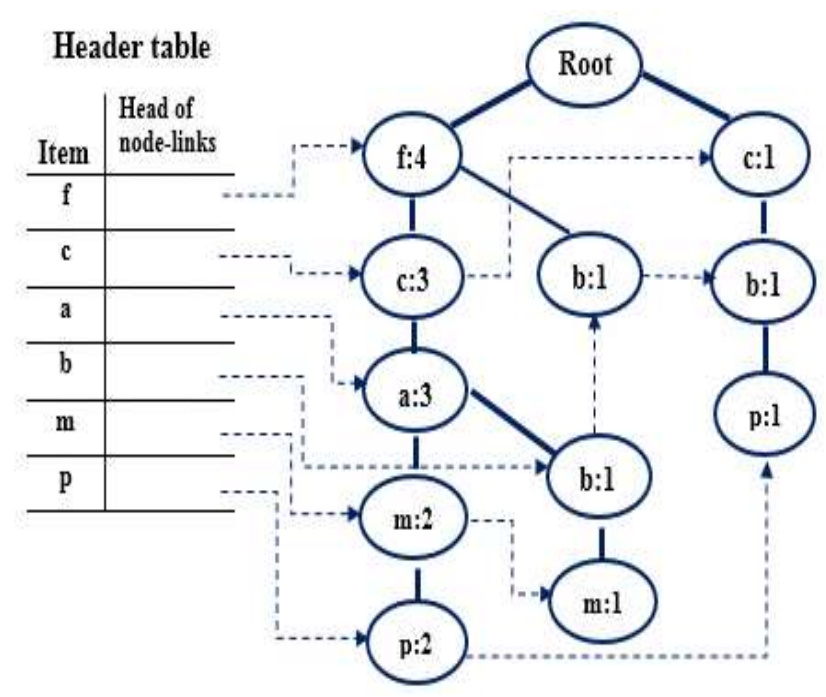

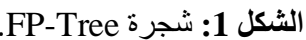

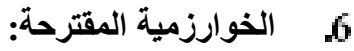

نقترح تطوير خوارزمية FP-growth من خلال استبدال بنية الشجرة بالمخطط الموجه، حيث تتألف الخوارزمية المقترحة (Graph Frequent) Patteren GFP-growth)

1 بناء جدول العناصر المتكررة H-table.

2. بناء المخطط الموجه FP-Graph.

3. التنقيب عن العناصر أو النماذج المتكررة. والثكل 2 يمثل المخطط التدفقي الذي يمثل الخطو ات السنابقة.

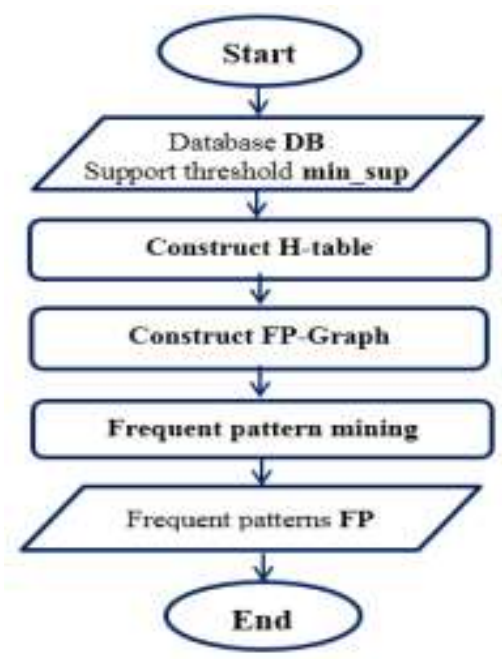

الثكل 2: المخطط التدفقي للخوارزمية المقترحة. 


\subsubsection{IF ItemNodePointer $==$ null.}

\subsubsection{Construct new FP-Graph node.}

2.3.4 Insert the ItemNodePointer address into $\mathrm{H}$ Table;

/* Assume that the ItemNodePointer is filled at this stage. $* /$

/* Adjust ParentNodePointer and TransId for tagging pattern-path with transId */

IF Parent-list! = null/* this current item-identifier node has parent $* /$

ParentNodePointer=Add Prefix Pattern Parent Node.

\section{Else}

Construct empty Parent-list and then Add Prefix

Pattern Parent Node in it.

3.3 Add this TransId to TransId-list.

Frequent itemset: التنقيب عن العناصر المتكررة

6.3

بعد بناء المخطط بطريقة مضغوطة، تتوقف عملية مسح قاعدة البيانات، ليتم

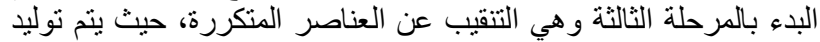

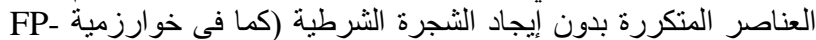

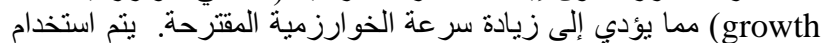

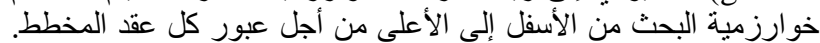

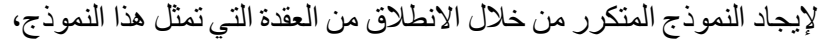

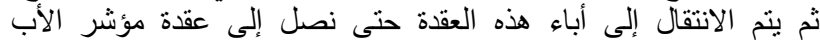

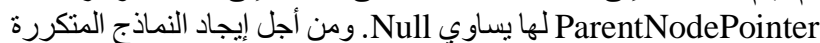

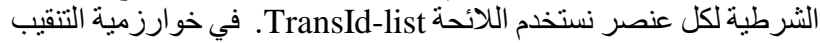
عن العناصر المتكررة، يتم الوصول إلى ParentNodePointer من القائمة Parent-list TransId-list الثرطية. بعد توليد النماذج الثرطية CP يتم حساب قيمة الدعم لها من أجل لئل

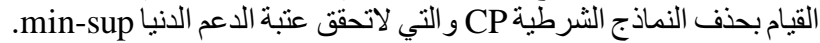
لينت بعدها إيجاد مجمو عات النماذج المتكررة المتاحة النهائية النية. و المخطط التدفقي المعبر عن خوارزمية التنقيب عن العناصر المتكررة موضح في الثكل 4.
فقط معرفة العقد الآباء لعقدة هذا النموذج ويتم تخزينها في قائمة-Parent)

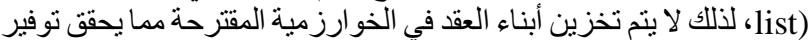
بالزمن و المساحة التخزينية.

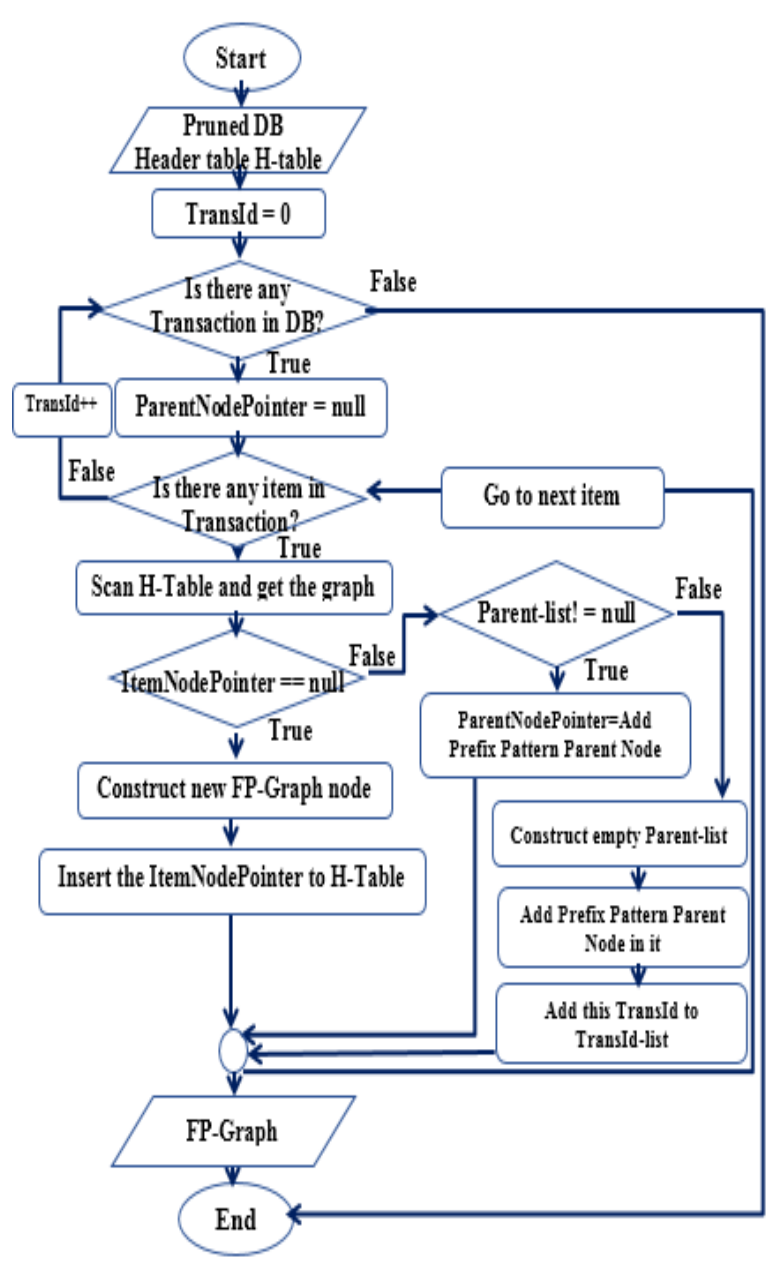

الثكل 3: المخطط التدفقي لبناء FP-Graph.

أما الثيفرة الز ائفة لبناء المخطط موضحة في الجدول 2.

الجدول 2: الثيفرة الزائفة لبناء المخطط.

Algoithm1: Procedure of Construct FP-Graph.

Input: A transaction database DB and frequent item header table $\mathbf{H}$-table.

\section{Output: FP-Graph.}

Initialize TransId to 0 .

For each Transaction $\mathrm{t} \in \mathrm{DB}$.

2.1 Initialize ParentNodePointer $=$ null.

2.2 transId++;

2.3 For each item $i \in t$.

2.3.1 ItemNodePointer $=$ scan $\mathrm{H}$-Table and get the graph pointer. 
مثال توضيحي: سنقوم بتطبيق الخوارزمية المقترحة على قاعدة المعطيات المبات

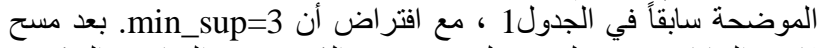

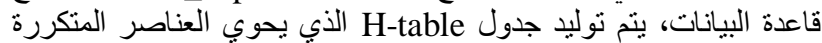

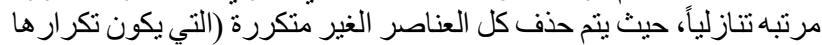

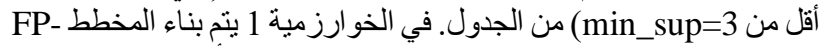
Graph

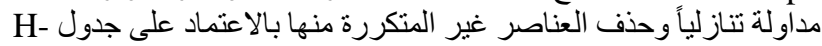

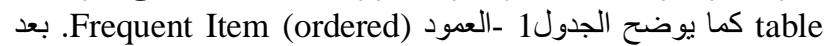

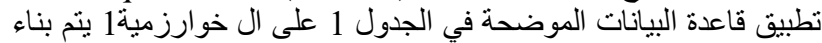
FP-Graph

كل المداو لات يتم تمثيلها من خلال Parent-list و التي تحوي مؤشرات للعقد

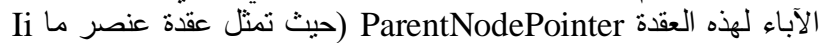

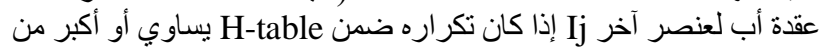

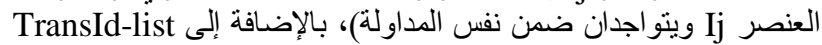
التي تحوي أرقام المداولات والتي والتي تستخدم من أجل تعليم (Tagging)

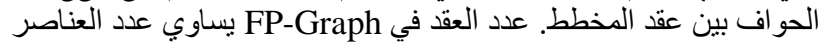

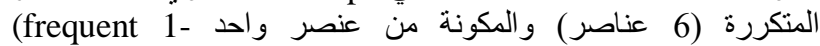
itemsets)

في المثال السابق يحوي المخطط ست عقد كما هو موضح في الثكل 5.

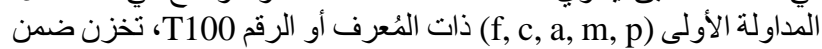

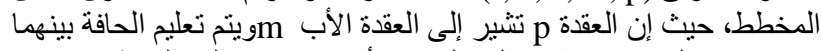

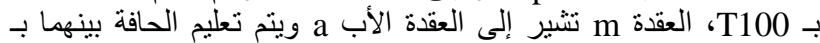

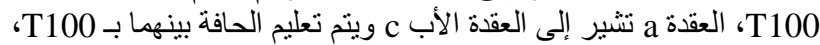

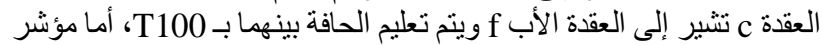

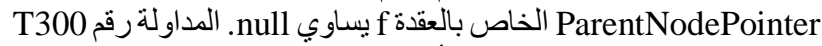

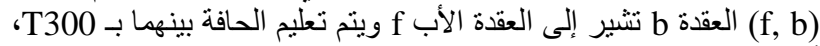

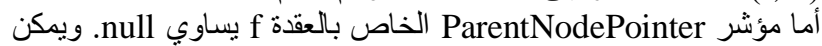

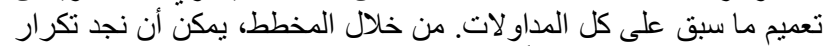
أي عنصرين منكررين، مثلاً

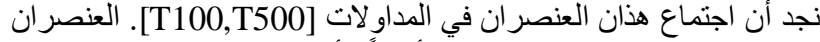

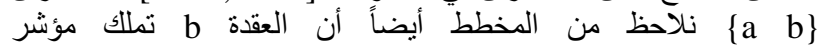
ParentNodePointer

يتم تطبيق الخوارزمية 2 على المخطط الذي تم بناؤه في المرحلة السابقة

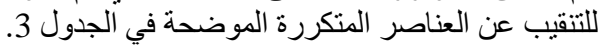

تبدأ عملية التنقيب من العنصر الموجود أسفل الجدول H-table، وبنطبيق

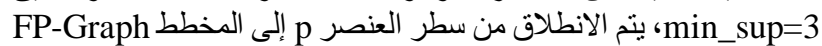

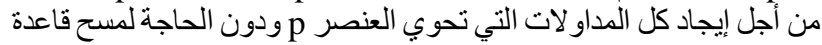

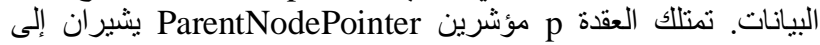

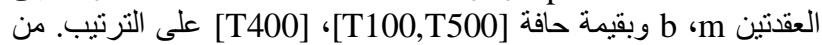

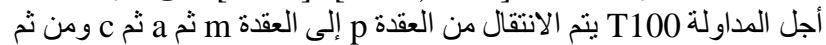

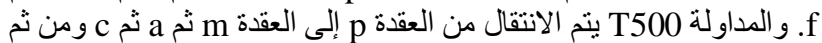
f

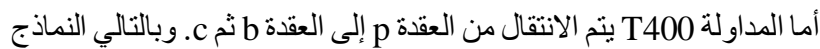

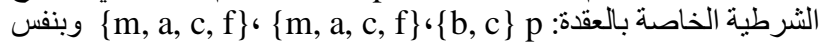

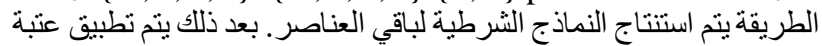

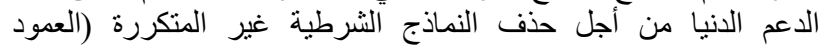
الأيp conditional patterns based on min_sup

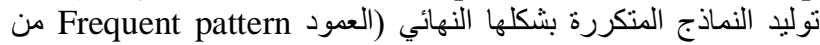
الجدول 4) من خلال إيجاد كل المجمو عات المئكية المكنة من النماذج الثرطية

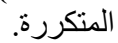

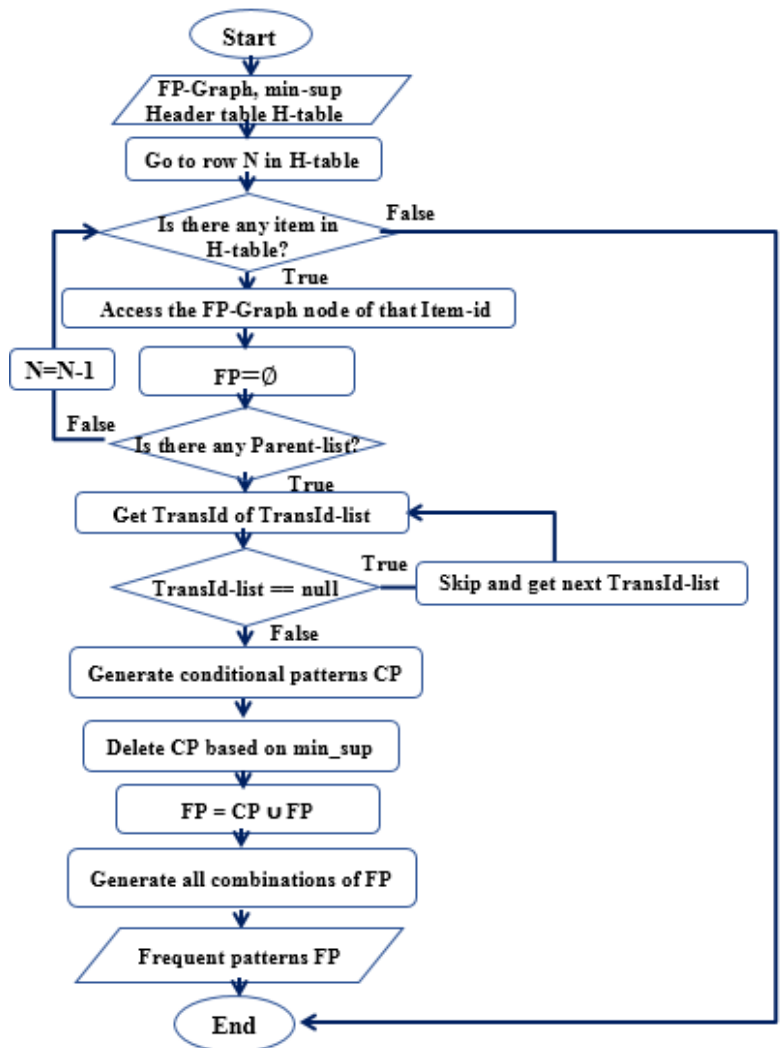

Frequent الثكل 4: المخطط التدفقي للتنقيب عن العناصر المتكررة .patterns

والثيفرة الزائفة لخوارزمية التنقيب عن العناصر المتكررة موضحة في الجدول

الجدول 3: الثيفرة الزائفة للتنقيب عن العناصر المتكررة.

Algorithm2: Procedure of Frequent itemset mining.

Input: FP-Graph, Support threshold min_sup and frequent item header table $\mathrm{H}$-table.

Output: Frequent patterns FP.

For (each bottom up item from H-table).

Access the FP-Graph node of that Item-id.

$$
1.2 \mathrm{FP}=\{\varnothing\} .
$$

1.3 For (each Parent-list).

1.3.1 Get TransId of TransId-list.

1.3.2 IF (TransId-list is empty).

1.3.3 Skip and get next TransId-list.

1.3.4 For (each TransId in TransId-list).

1.3.5 Generate conditional patterns CP.

1.3.6 Delete CP based on min_sup.

$$
\text { 1.3.7 FP = CP } \cup \mathrm{FP} .
$$

1.3.8 Generate all combinations of FP. 


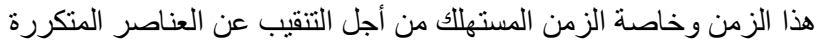

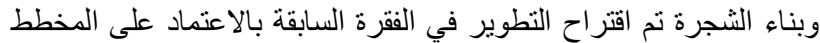
الموجه كما تم شرحة.

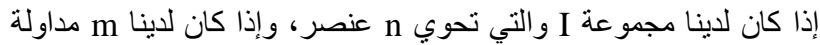

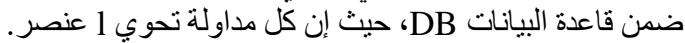

أفضل حالة: درجة تعقيد الخوارزمية من أجل التنقيب عن العناصر المتكررة

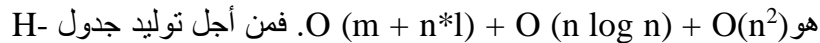

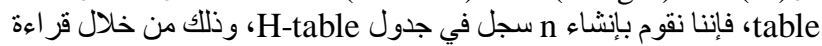

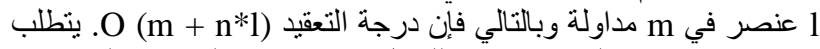

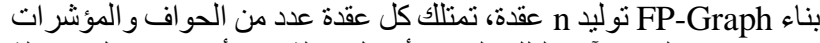

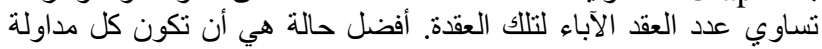

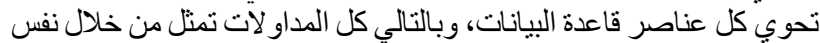

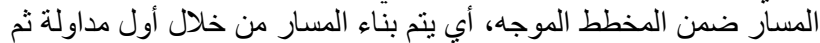
ينم تعديل لائحة TransId-list فقط في باقي المداولات. وبالتالي درالي درجة
الجدول 4: النماذج المتكررة Frequent pattern .

\begin{tabular}{|c|c|c|}
\hline $\begin{array}{l}\text { Conditional } \\
\text { patterns }\end{array}$ & $\begin{array}{l}\text { conditional } \\
\text { patterns } \\
\text { based on } \\
\text { min_sup }\end{array}$ & Frequent pattern \\
\hline $\begin{array}{l}\mathrm{p}:\{\mathrm{m}, \mathrm{a}, \mathrm{c}, \mathrm{f}\} \mid\{\mathrm{b}, \\
\mathrm{c}\} \mid\{\mathrm{m}, \mathrm{a}, \mathrm{c}, \mathrm{f}\}\end{array}$ & $\mathrm{p}:\{\mathrm{c}\}$ & $\{p\},\{p, c\}$ \\
\hline $\begin{array}{c}\mathrm{m}:\{\mathrm{a}, \mathrm{c}, \mathrm{f}\} \mid \\
\{\mathrm{b}, \mathrm{a}, \mathrm{c}, \mathrm{f}\} \mid \\
\{\mathrm{a}, \mathrm{c}, \mathrm{f}\}\end{array}$ & $m:\{a, c, f\}$ & $\begin{array}{c}\{\mathrm{m}\},\{\mathrm{m}, \mathrm{a}\},\{\mathrm{m}, \mathrm{c} \\
\},\{\mathrm{m}, \mathrm{f}\},\{\mathrm{m}, \mathrm{a}, \\
\mathrm{c}\},\{\mathrm{m}, \mathrm{a}, \mathrm{f}\},\{\mathrm{m}, \mathrm{c}, \\
\mathrm{f}\},\{\mathrm{m}, \mathrm{a}, \mathrm{c}, \mathrm{f}\}\end{array}$ \\
\hline $\begin{array}{c}\mathrm{b}:\{\mathrm{c}\}|\{\mathrm{f}\}|\{\mathrm{a}, \mathrm{c}, \\
\mathrm{f}\}\end{array}$ & $\mathrm{b}:\{\}$ & $\{b\}$ \\
\hline $\begin{array}{c}\mathrm{a}:\{\mathrm{c}, \mathrm{f}\}|\{\mathrm{c}, \mathrm{f}\}| \\
\{\mathrm{c}, \mathrm{f}\}\end{array}$ & $\mathrm{a}:\{c, f\}$ & $\begin{array}{c}\{a\},\{a, c, f\},\{a, c\} \\
\{a, f\}\end{array}$ \\
\hline $\mathrm{c}:\{f\}|\{f\}|\{f\}$ & $c:\{f\}$ & $\{\mathrm{c}\},\{\mathrm{c}, \mathrm{f}\}$ \\
\hline$f:\{\}$ & $f:\{\}$ & $\{\mathrm{f}\}$ \\
\hline
\end{tabular}

6.4

إن نسبة كبيرة من زمن التنفيذ خو ارزمية FP-growth الأساسية بستهلك في

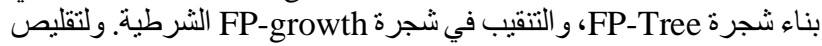

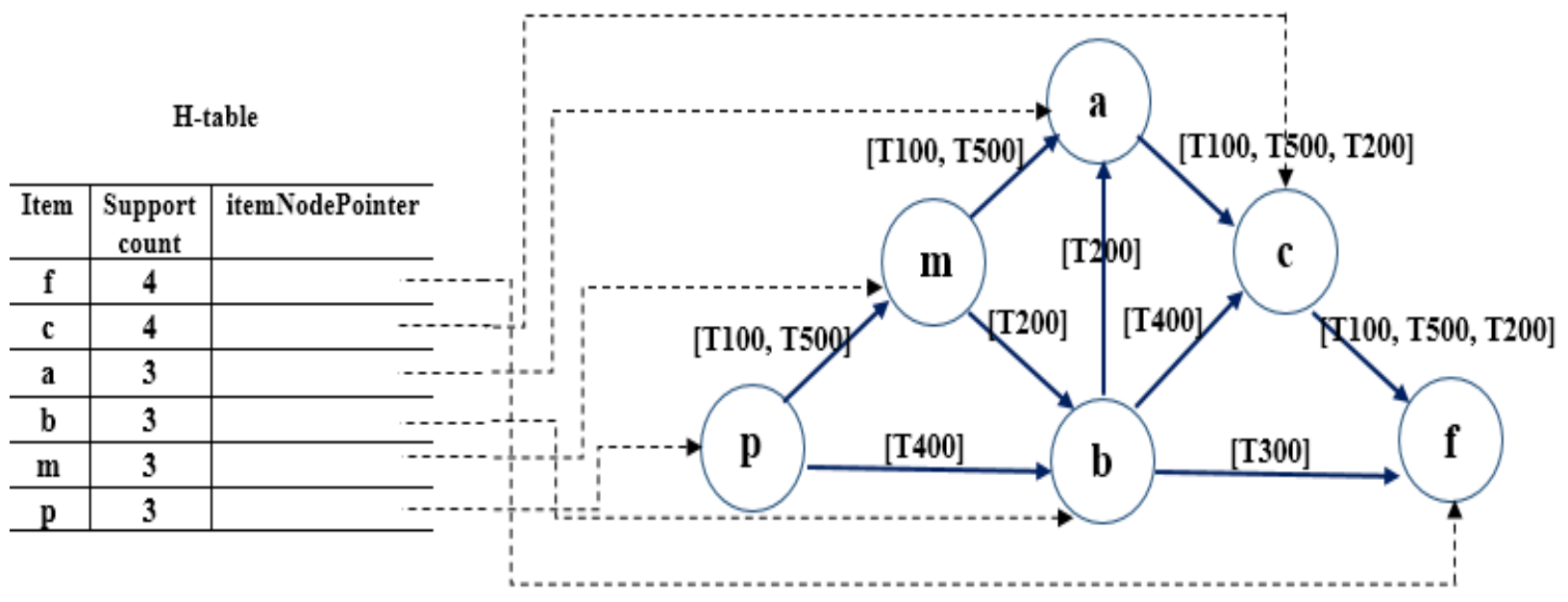

الشكل 5: مخطط FP-Graph لقاعدة البيانات السابقة.

لزيارة كل عقدة واضافة مؤشر للعقدة الأب الخاص بها وبالتالي درجة التعقبد $. \mathrm{O}(\mathrm{n}(\mathrm{n}-1))=\mathrm{O}\left(\mathrm{n}^{2}\right)$

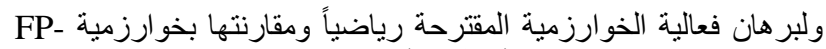

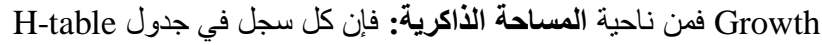

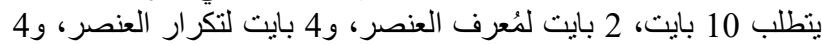

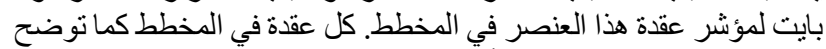

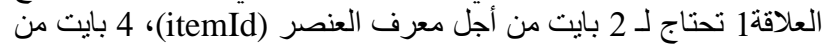

التعقيد (n log n) (أما عند التنقيب عن النماذج المنكررة، فإذا كان لدينا

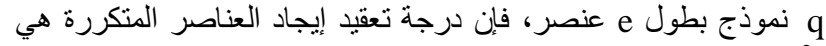
$\mathrm{O}\left(\mathrm{q}^{*} \mathrm{e}\right)=\mathrm{O}\left(\mathrm{n}^{2}\right)$

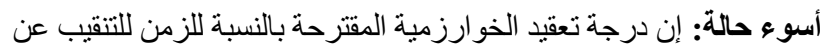
العناصر المنكررة هو إن درة

في هذه الحالة الاختلاف فقط في مرحلة بناء المخطط الموجه، يتم تمثيل كل

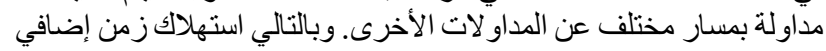


FP- الخوارزمية المقترحة أسرع بمقدار 60\% تقريباً من وخوارزمية Growth

\section{7. تطبيق الخوارزمية المقترحة تفرعياً:}

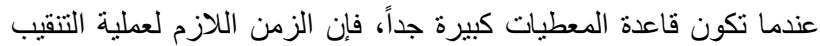

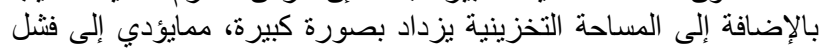

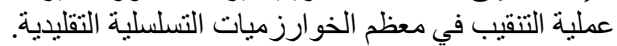

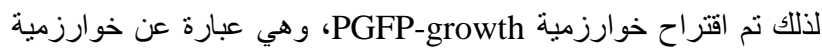

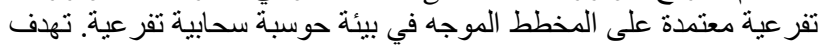

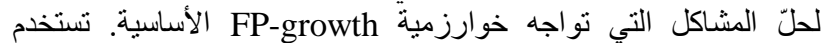
المخطط الموجه من أجل توفير المساحة التخزينية اللازمة لتهنة لتخزين قاعدة

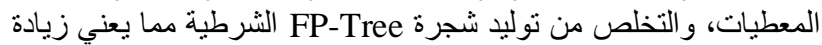

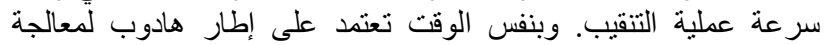

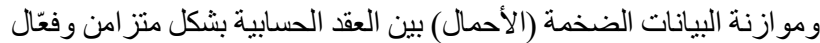
وموزع من خلال البنية Map Reduce.

يعتبر هادوب برنامج مفتوح المصدر بإطار تخزين موز ع لمجمو عات كبيرة

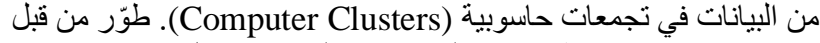

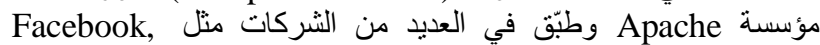
Yahoo, Amazon, IBM

يتميز بالعديد من الخصائص مثل انخفاض الكلفة المادية، حيث يمكنتا بناء إنهاء

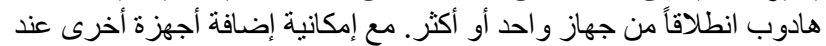

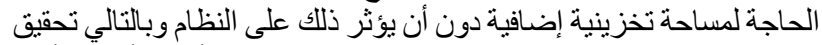

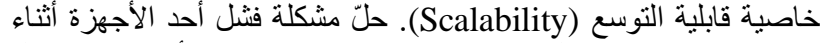

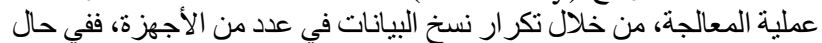

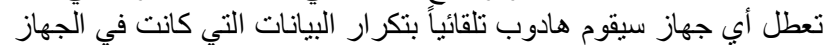

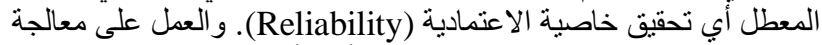

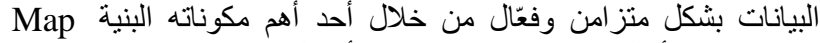
Reduce

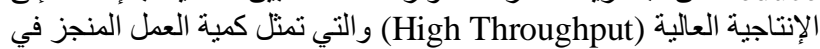

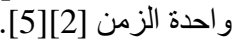

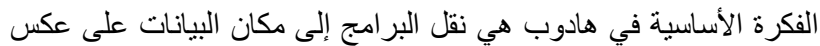

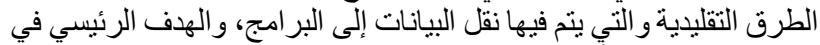

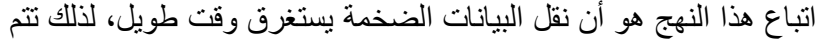
معالجتها في مكانها.

أي أن معظم عمليات المعالجة تجري في محطات أو عقد البيانات Data) حode)

$$
\text { يتكون هادوب من ثلاث مكونات أساسية: }
$$

1 الملفات المستخدم لتخزين البيانات.

MapReduce المتوازية في معالجة البيانات.

(Yet Another Resource Negotiator) YARN المسؤول عن إدارة التجمعات (Cluster).

الاعتماد الأساسي للهادوب في عملية المعالجة على تقنية Map وتقنية Reduce

1. تقوم map بتقسيم البيانات إلى أجز اء (مفتاح Key / قيمة Value). 2. ترسل مخرجات map إلى مرحلة Shuffle لترتيبها.
أجل Parent-list، 4 بايت من أجل TransId-list، حيث N: مثلث عدد

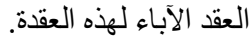

$$
\text { Space complexity (vertix) }=2+4 N+4 N
$$

$$
=2+8 N
$$

وبالتالي لتوضيح فعالية البنية المقترحة FP-Graph ومدى ضغطئه المبا لقاعدة المعطيات، سوف نقوم بحساب المساحة التخزينية المطلوبة للمبة للمثنال السابق،

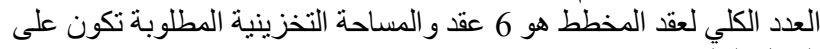

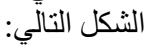

$$
\text { المساحة المطلوبة لجدول H-table: } 10 \text { =6= } 60 \text { بايت. }
$$

المساحة المطلوبة للمخطط FP-Graph: كل عقدة تتطلب مساحة تخزينية

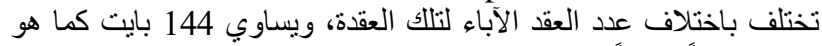

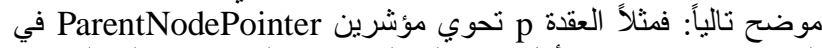

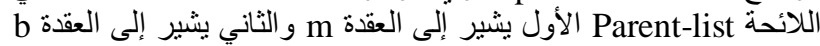

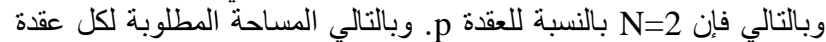

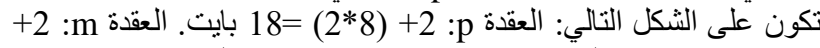

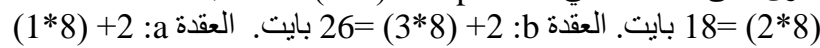

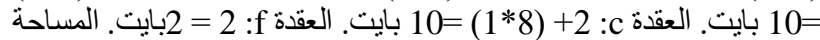
الكلية المطلوبة لتخزين العقد = 84 بايت. المباحة العنة الكلية المطلوبة لتخزين

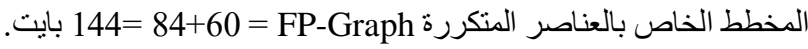

بالمقارنة بين الخوارزمية المقترحة وخو ارزمية FP-growth من حيث عدد المدابث

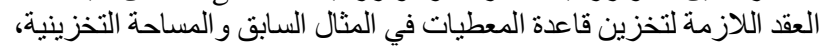

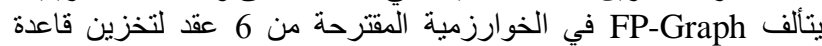

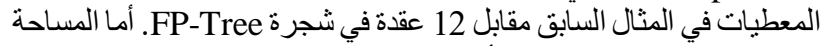
التخزينية المطلوبة لتخزين من أجل 144 FP-Graph بايت مقابل 292 بايت البن

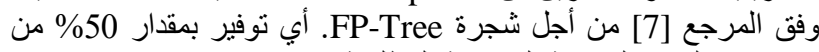
حيث عدد العقد و المساحة التخزينية المطلوبة المنة

أما من حيث الزمن: يعتمد الزمن الذي تستغرقه الخوارزمية المقترحة على فلى ثلاثة أزمنة رئيسية كما هو موضنح في الزئن العلاقة2:

Time complexity $=n * T 1+m * T 2+r * T 3$

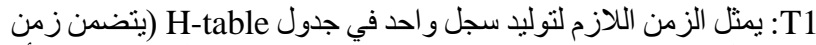

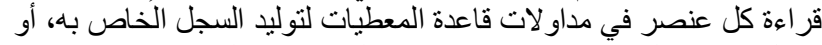

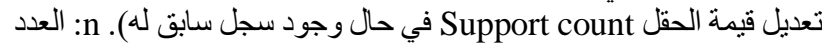

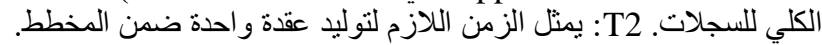

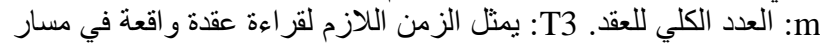

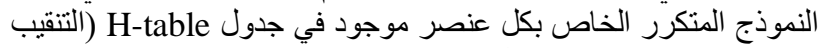

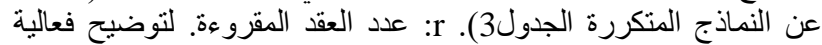

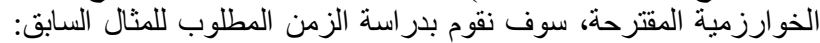

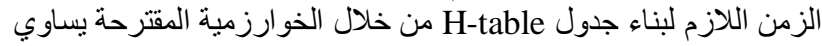
و 16* T1

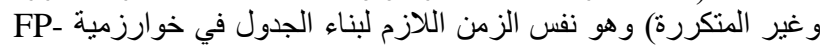

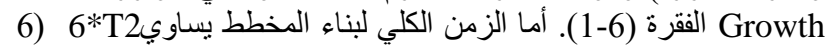

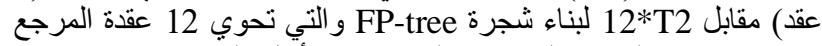

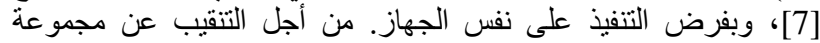

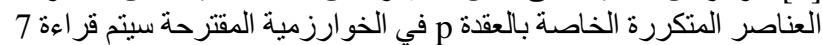

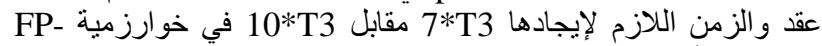

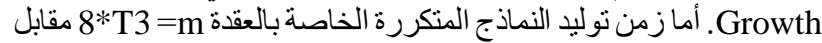

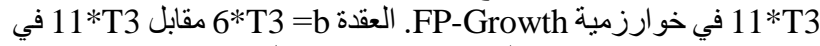

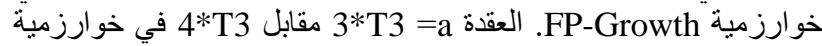
FP- العقدة FP-Growth

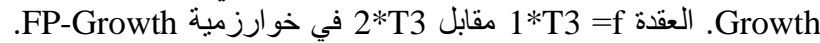

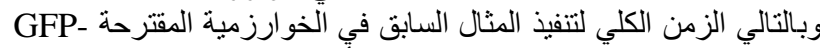

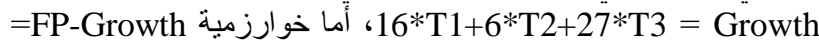
16*T1+12*T2+43*T3 بالمقارنة بين زمن تنفيذ الخوارزميتان نجد أن 
ومنها إلى البنية reduce و التي تعمل على تجميع نتائج المرحلة السابقة،

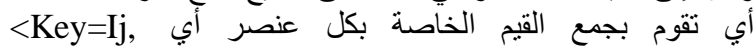

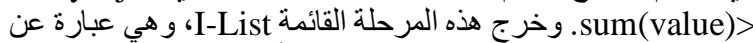

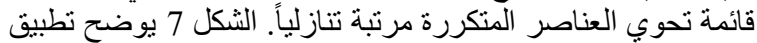

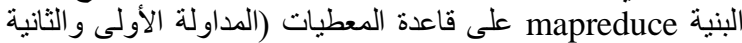
ل للسهولة) في المثال السابق.

تجميع العناصر المتكررة: في البحث [14] يتم تقسيم القائمة I-List

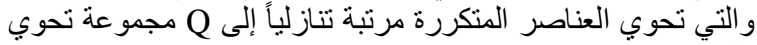

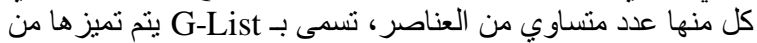

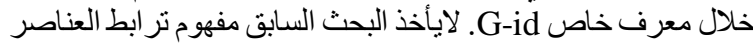

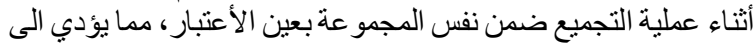

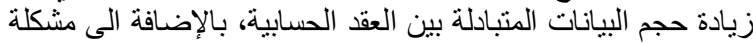
عدم التوازن في الأحمال بين العقد الحسابية (Imbalanced load).

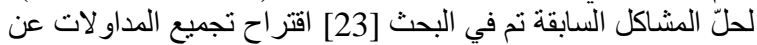

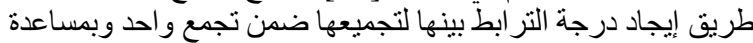

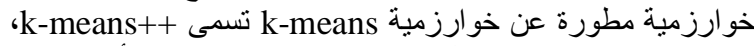

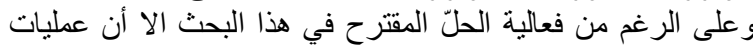

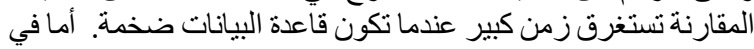

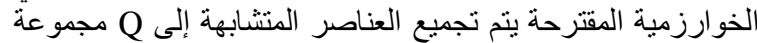

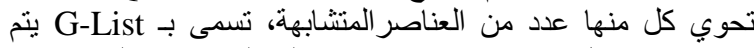

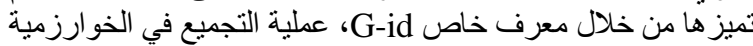

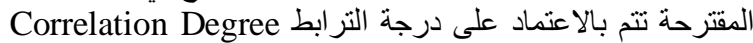

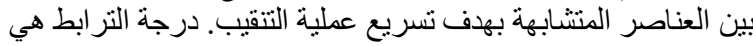

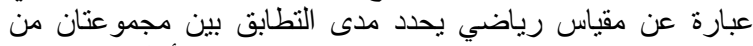

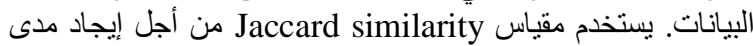

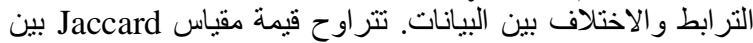

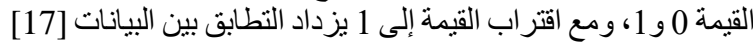

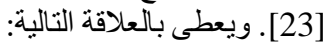

$$
\operatorname{Jaccard}\left(\mathrm{I}_{\mathrm{i}}, \mathrm{I}_{\mathrm{j}}\right)=\frac{I i \cap I j}{I i U I j}
$$

حيث يتم تجميع العناصر المتشابهة ضمن مجمو عة واحدة تسمى بـ G-List

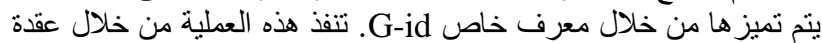

$$
\text { حسابية و احدة. }
$$

4. تطبيق خوارزمية GFP-growth بالشكل التفرعي من أجل توليد

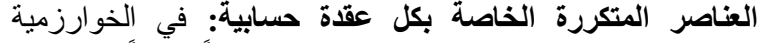

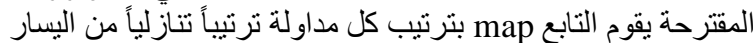

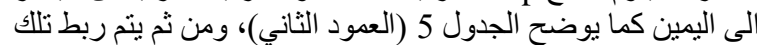

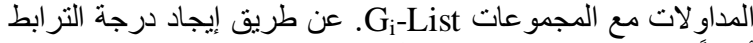

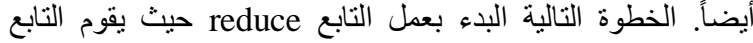

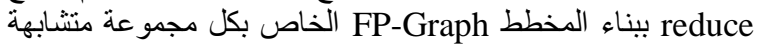

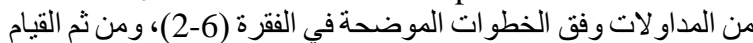

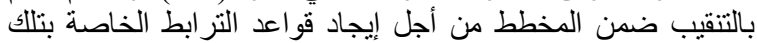

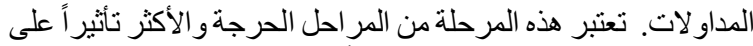

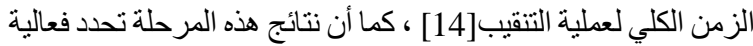

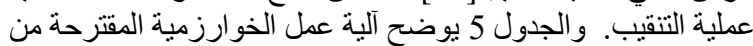

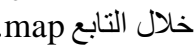

\begin{tabular}{|c|c|c|}
\hline $\begin{array}{l}\text { Map inputs } \\
\text { (transactions) } \\
\text { Key=""':value }\end{array}$ & $\begin{array}{l}\text { (ordered) Frequent } \\
\text { Item }\end{array}$ & $\begin{array}{c}\text { Map outputs } \\
\text { (transactions) } \\
\text { Key: value }\end{array}$ \\
\hline T100: & $\mathrm{fcamp}$ & $\mathrm{p}: \mathrm{m}$ a c $\mathrm{f}$ \\
\hline
\end{tabular}

الجدول 5: عل التابع map في الخوارزمية PGFP-growth.
3. تقوم reduce بأخذ مخرجات المرحلة السابقة كمدخلات لتجميعها

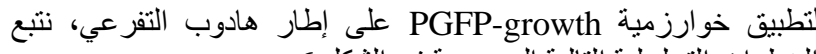
الخطوات التسلسلية التالية الموضحة في الثكل 6:

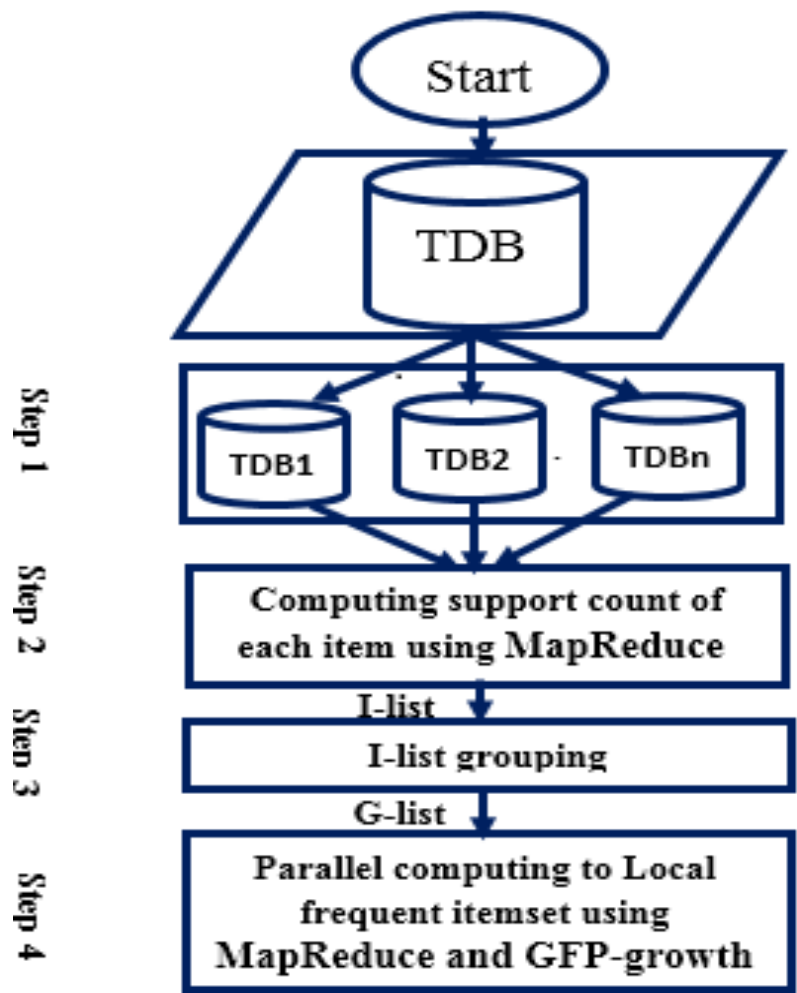

Local frequent itemset

䄈

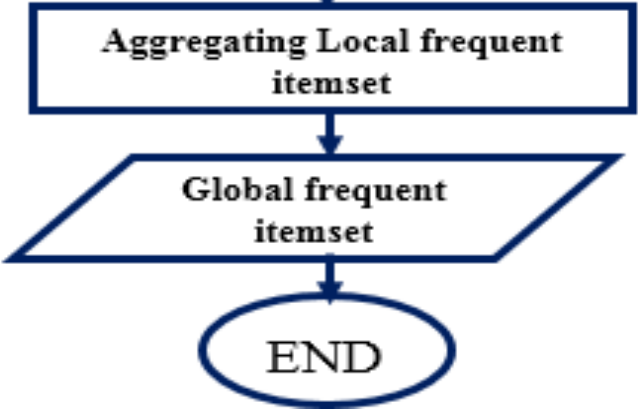

الثكل 6: المخطط التدفقي للخوارزمية التفر عية المقترحة -PGFP .growth

تقسيم قاعدة المعطيات: في هذه المرحلة يتم تجزئة قاعدة المعطيات

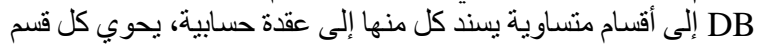

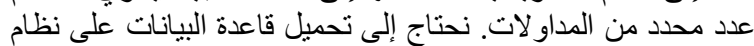

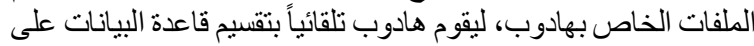
العقد الحسابية بشكل متساوي.

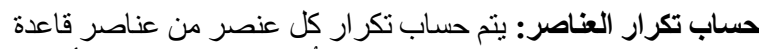

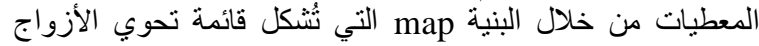

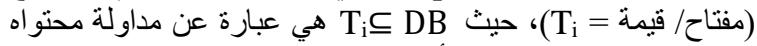

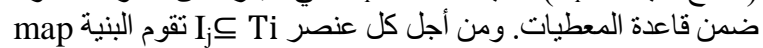

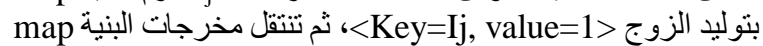
إلى المرحلة shuffle التي تقوم بترنيبها حسب المفتاح (العنصر)، 


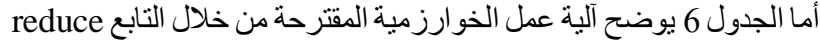

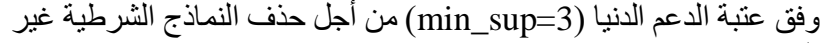
المتكررة.

الجدول 6: عمل التابع Reduce في الخوارزمية PGFP-growth.

\begin{tabular}{|c|c|}
\hline $\begin{array}{c}\text { Reduce inputs } \\
\text { (transactions) } \\
\text { Key: value }\end{array}$ & $\begin{array}{c}\text { Reduce outputs } \\
\text { Frequent conditional } \\
\text { patterns based on } \\
\text { min_sup }\end{array}$ \\
\hline $\mathrm{p}:\{\mathrm{m} \mathrm{a} \mathrm{c} \mathrm{f/b} \mathrm{c} \mathrm{/} \mathrm{m} \mathrm{a} \mathrm{c} \mathrm{f}\}$ & $\{(\mathrm{c}: 3)\} \mid \mathrm{p}$ \\
\hline $\mathrm{m}:\{\mathrm{a} \mathrm{c} \mathrm{f} / \mathrm{b}$ a c f / a c f $\}$ & $\{(\mathrm{a}: 3, \mathrm{c}: 3, \mathrm{f}: 3)\} \mid \mathrm{m}$ \\
\hline $\mathrm{b}:\{\mathrm{a}$ c f/ f/c $\}$ & \{\} $\mid \mathrm{b}$ \\
\hline $\mathrm{a}:\{\mathrm{c} \mathrm{f} / \mathrm{c} \mathrm{f} / \mathrm{c} f\}$ & $\{(\mathrm{c}: 3, \mathrm{f}: 3)\} \mid \mathrm{a}$ \\
\hline $\mathrm{c}:\{\mathrm{f} / \mathrm{f} / \mathrm{f}\}$ & $\{(\mathrm{f}: 3)\} \mid \mathrm{c}$ \\
\hline
\end{tabular}

5. إيجاد الخرج النهائي: من خلال تجميع نتائج كل العقد الحسابية الناتجة

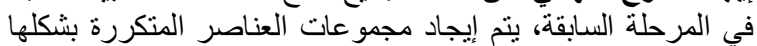
النهائي.

\begin{tabular}{|c|c|c|}
\hline facdg Imp & & $\begin{array}{c}\mathrm{m}: \mathrm{acf} \\
\mathrm{a}: \mathrm{cf} \\
\mathrm{c}: \mathrm{f}\end{array}$ \\
\hline $\begin{array}{c}\text { T200: } \\
\text { a b c f } 1 \mathrm{mo}\end{array}$ & $\mathrm{fcab}$ & $\begin{array}{c}\text { m: b a c f } \\
\text { b: a c f } \\
\text { a: c f } \\
\text { c: f }\end{array}$ \\
\hline $\begin{array}{c}\text { T300: } \\
\text { b f h j o }\end{array}$ & $\mathrm{fb}$ & $\mathrm{b}: \mathrm{f}$ \\
\hline $\begin{array}{c}\text { T400: } \\
\text { b c k s p }\end{array}$ & $\mathrm{c} \mathrm{b} \mathrm{p}$ & $\begin{array}{c}\text { p: b c } \\
b: c\end{array}$ \\
\hline $\begin{array}{c}\text { T500: } \\
\text { a f c e l p m n }\end{array}$ & $\mathrm{fcamp}$ & $\begin{array}{c}\mathrm{p}: \mathrm{m} \text { a c f } \\
\mathrm{m}: \text { a c f } \\
\text { a: c f } \\
\text { c:f }\end{array}$ \\
\hline
\end{tabular}

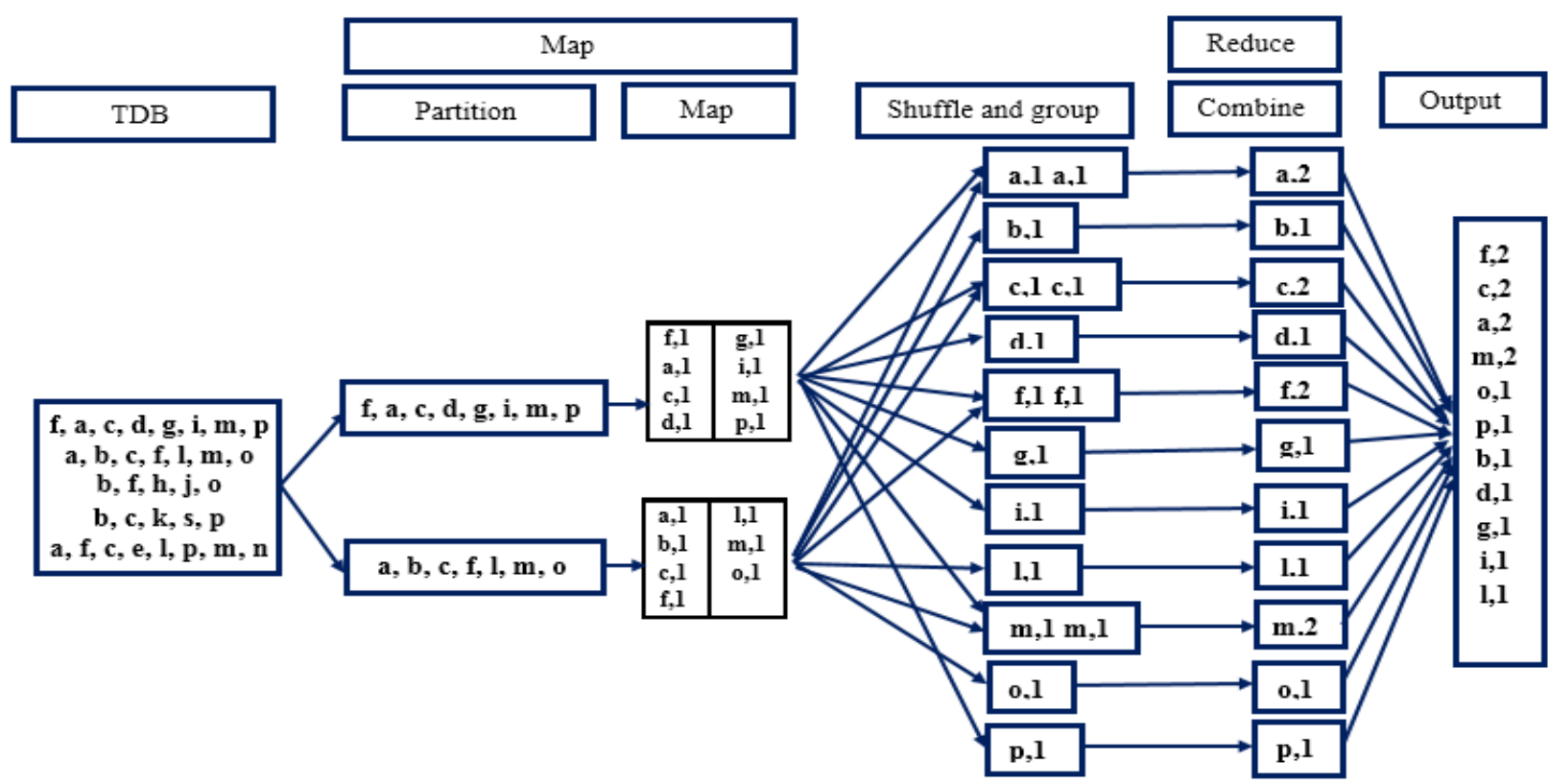

الثكل 7: تطبيق البنية MapReduce على قاعدة المعطيات السابقة (المداولة الأولى و الثانية فقط للتبسيط).

Intel®Core ${ }^{\mathrm{TM}} \mathrm{i} 32.30 \mathrm{GH}_{\mathrm{z}}$ ومعالج، Ubuntu 16.4 LTS-64Bit

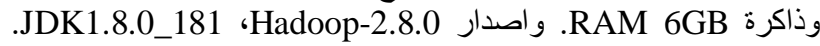

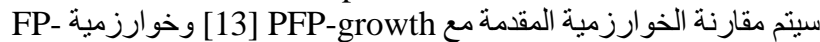

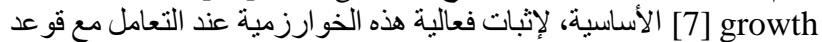
البيانات الضخمة، وتوفير الزمن اللازم لعملية التنقيب والمئية المساحة التخزينية المطلوبة، وتخفيض التعقيد الحسابي و الاتصال بين عقد التجمع، وذللك عند التيل
النتائج التجريبية: - 2 - 2 - 2

8

تم بناء خوارزمية PGFP-growth المقترحة بلغة الـ Java وبيئة

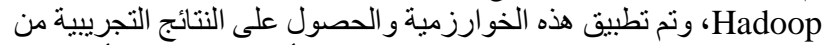

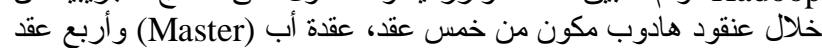
أبناء (Slaves). كل العقد نمتلك نفس البنية البرمجية و المادية، نظام التشغيل 


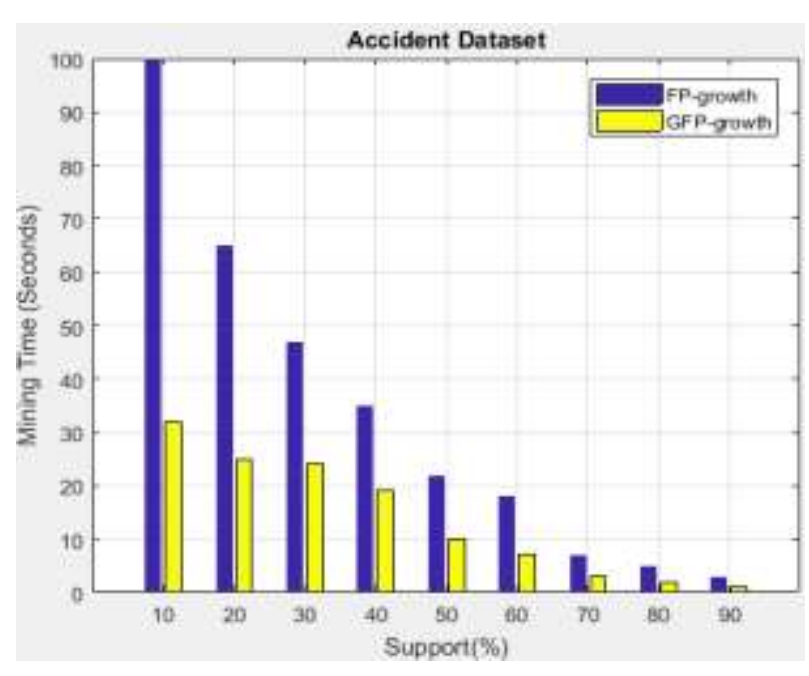

الشكل 9: زمن التنفيذ من أجل عنبات دعم مختلفة (Accident).

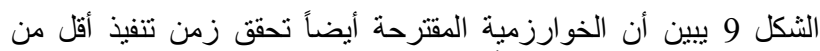

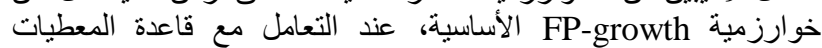
Accidents

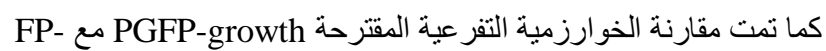

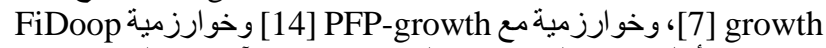

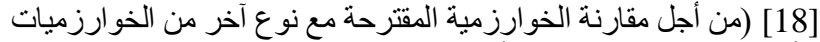

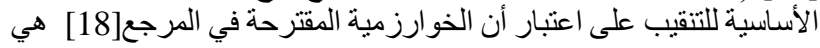

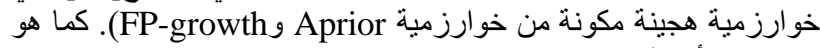

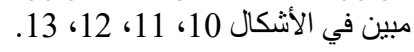

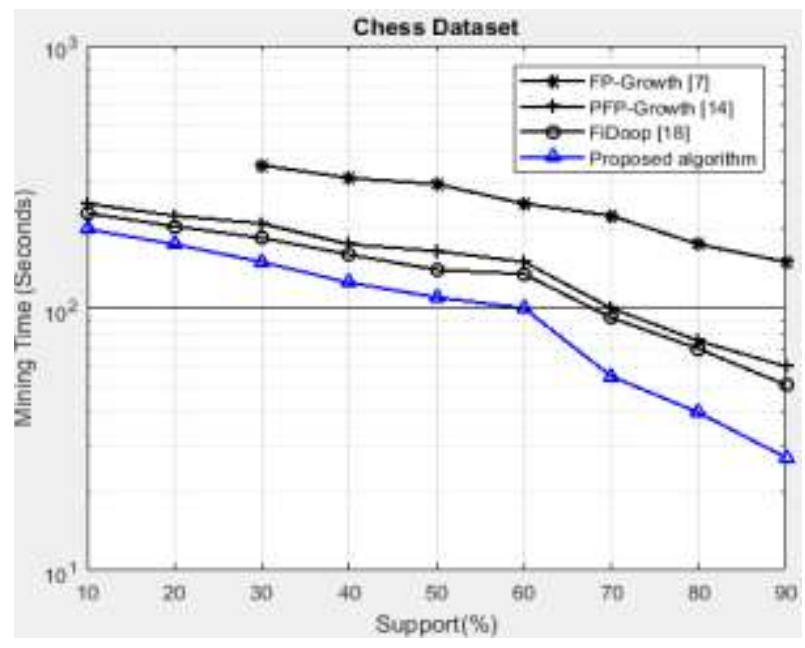

الثكل 10: زمن التنفيذ من أجل عنبات دعم مختلفة (Chess).
تطبيق عتبات min-sup مختلفة. استخدمت مجموعة من قواعد معطيات

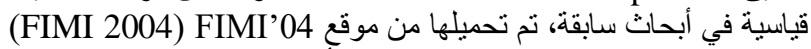

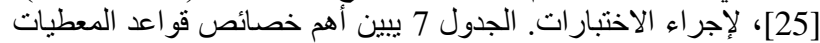

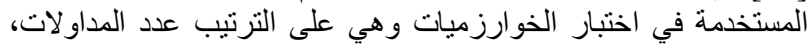
متوسط طول المداولة، عدد العناصر ، الحزئ الحهي.

الجدول 7: خصائص قو اعد المعطيات المستخدمة.

\begin{tabular}{|c|c|c|c|c|}
\hline Dataset & Trans & $\begin{array}{c}\text { Avg. } \\
\text { Length }\end{array}$ & Items & $\begin{array}{c}\text { Size } \\
(\mathrm{MB})\end{array}$ \\
\hline Chess & 3196 & 37 & 75 & 0.34 \\
\hline Accident & 340183 & 34 & 468 & 14.7 \\
\hline T40I10D100K & 100000 & 10 & 942 & 30.25 \\
\hline Kosarak & 990002 & 8 & 41270 & 40.5 \\
\hline
\end{tabular}

يوجد نوعان من قو اعد المعطيات: متفرقة (Sparse) أو كثيفة (Dense)، و هو مصطلح يستخدم في قو اعد البيانات متعددة الأبعاد لتحديد نسبة الخلايا

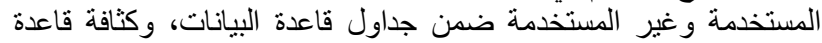

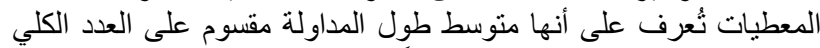

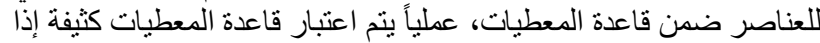
كانت نسبة الخلايا المستخدمة تزيد عن 10\% [25].

في البداية سوف يتم مقارنة النتائج التجريبية الخاصة بالخو ارزمية المقترحة المحسنة GFP-growth مع اليداية الأساسية و على عقدة حسابية واحدة لإثبات مدى فعالية الخو ارزمية المحسنة، باستخدام قاعدة المعطيات

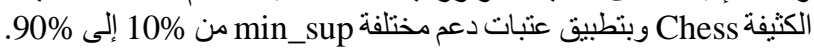

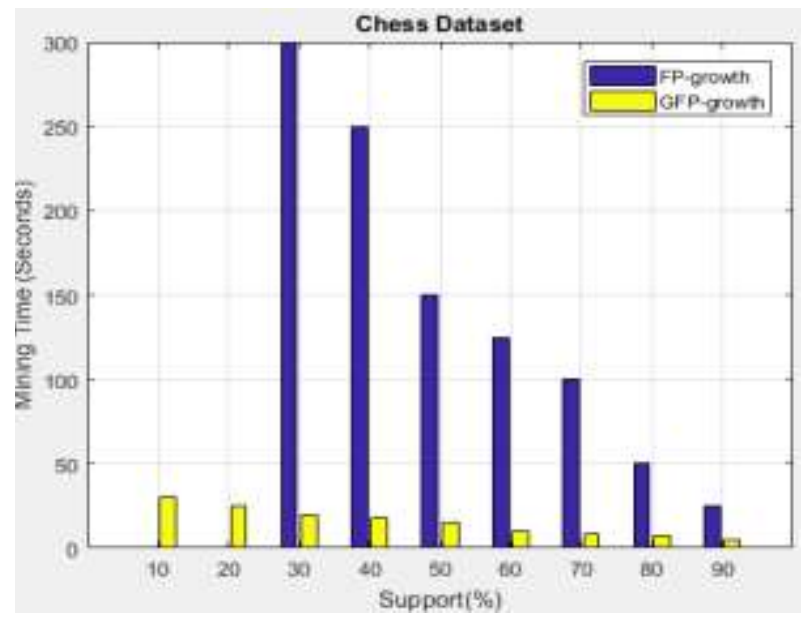

الشكل 8: زمن التنفيذ من أجل عتبات دعم مختلفة (Chess).

من الثكل 8 نجد أن الخوارزمية المقترحة تحقق زمن تنفيذ أسرع من النماف

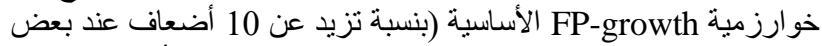

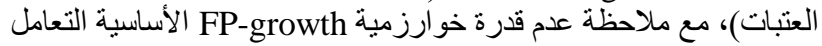

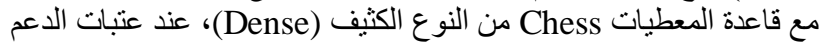
المنخفضة مثل 10\% و 20\% كما هو موضح بالثر الثنل. 


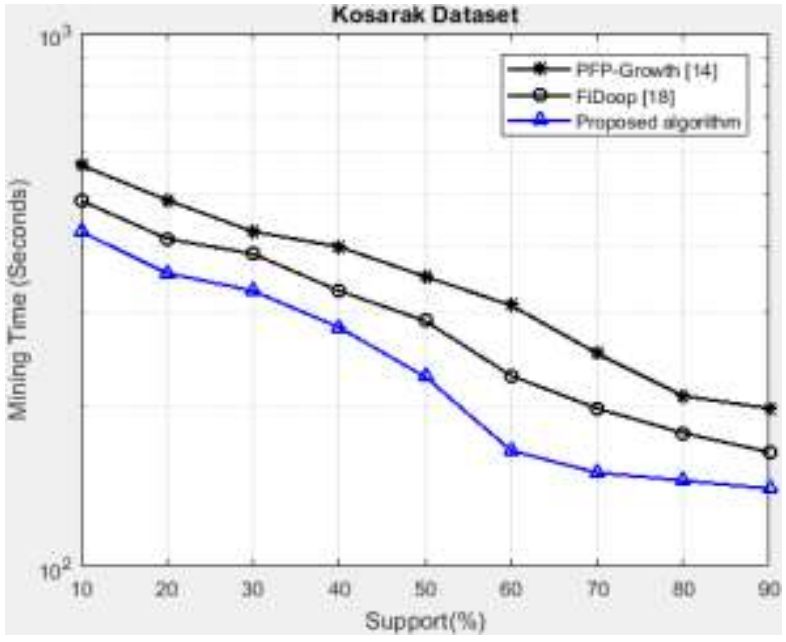

الشكل 13: زمن التنفيذ من أجل عتبات دعم مختلفة (Kosarak).

من الأشكال السابقة نجد أن الخوارزمية المقترحة تستغرق زمن تتفيذ أقل بين

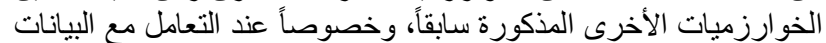

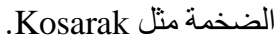

تم ضبط معامل الترابط عند تقسيم العناصر المتكررة بالقيم التالية ( 0.20 (0.60) (0.40, 0.60, 0.80, 1

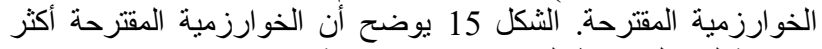

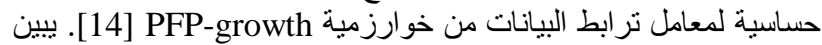

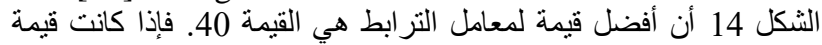

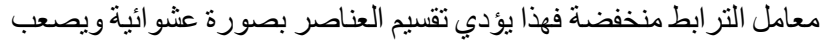
تقسيم العناصر المتكررة الى مجمو عات مستقلة عند ضبط فيط قيمة معامل التر ابط بقيمة كبيرة.

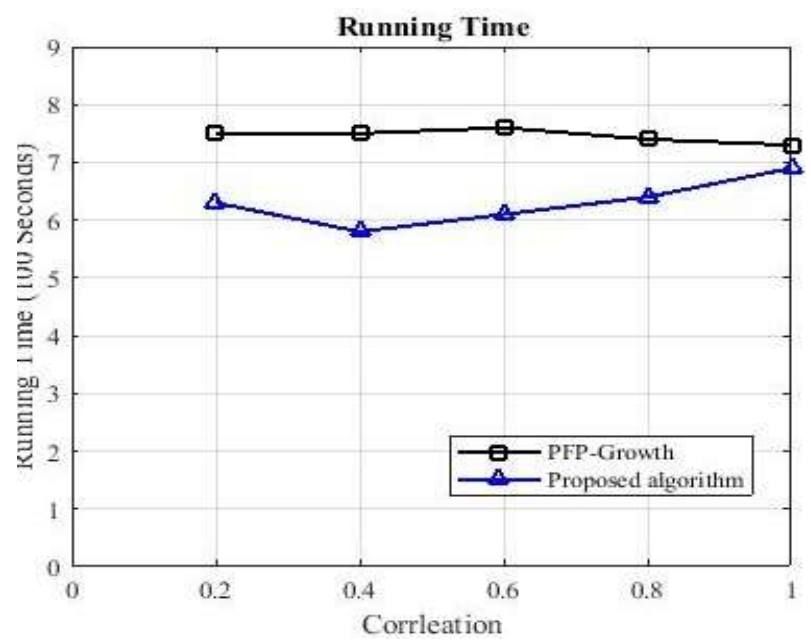

الثكل 14: تأثير معامل التر ابط على الخوارزمية المقترحة.

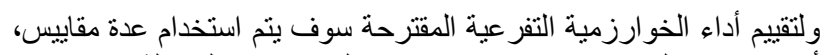

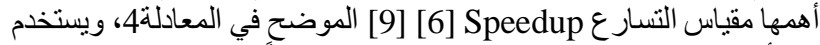

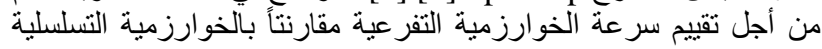
المطبقة على عقدة واحدة.

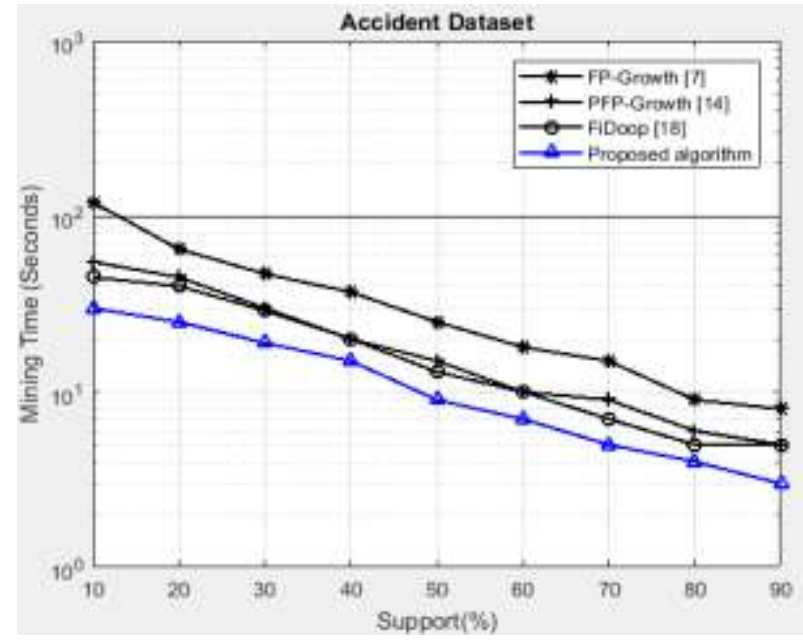

الثكل 11: زمن التنفيذ من أجل عتبات دعم مختلفة (Accident).

إن الخوارزمية المقترحة تحتاج زمن تتفيذ أقل للتنقيب عن العناصر المتكررة

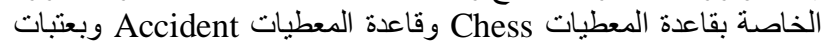

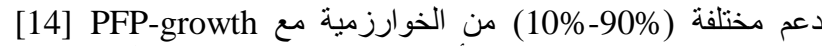
وخو ارزمية FP-growth [7] الأساسية كما هو موضح في فع الشكل 11 و12 (12. والثكل 13 يبين أن الخوارزمية المقترحة أيضاً تحقق زمن تنفيذ أسرع بين

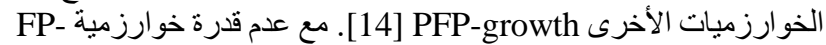

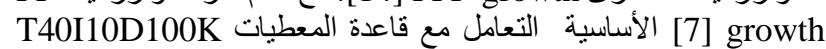
وقاعدة المعطيات Kosarak.

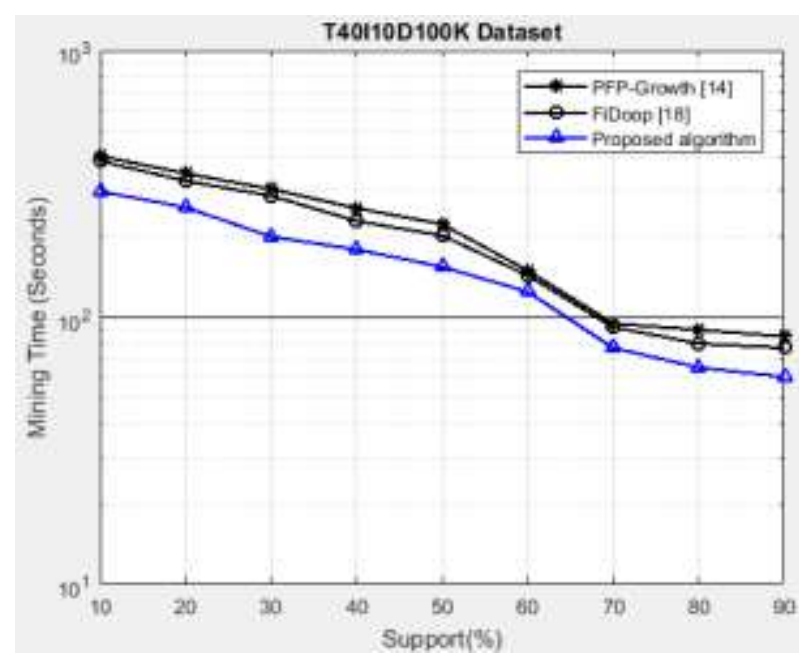

الثكل 12: زمن التنفيذ من أجل عتبات دعم مختلفة

(T40I10D100K)

Speedup $=\mathrm{S}(\mathrm{n})=\frac{T 1}{T n}$ 
الجدول8: زمن التتفيذ وقيم التسار ع Speedup بالاعتماد على قانون

Gustafson من أجل قاعدة البيانات (T40I10D100K).

\begin{tabular}{|c|c|c|c|c|c|}
\hline Problem size & \multicolumn{3}{|c|}{$\begin{array}{c}\text { Execution } \\
\text { Time(second) }\end{array}$} & \multicolumn{2}{|c|}{ Speedup } \\
\hline T40I10D100K & $\mathrm{T}_{1}$ & $\mathrm{~T}_{2}$ & $\mathrm{~T}_{4}$ & $S(2)$ & $\mathrm{S}(4)$ \\
\hline 30 & 466 & 241 & 118 & 1.93 & 3.94 \\
\hline 60 & 1020 & 511 & 257 & 1.99 & 3.96 \\
\hline 120 & 2134 & 1072 & 535 & 1.99 & 3.98 \\
\hline
\end{tabular}

من الجدول 8 نجد أنه عندما تم معالجة قاعدة البيانات T40I10D100K

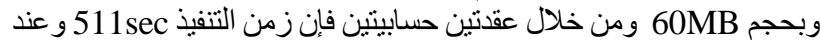

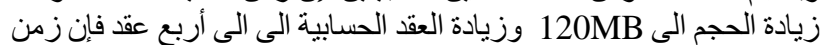

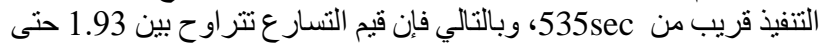

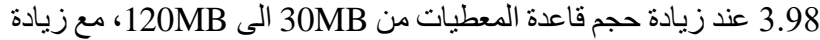
عدد العقد الحسابية من 2 حتى 4.98.

الجدول 9: زمن التنفيذ وقيم التسار ع Speedup بالاعتماد على قانون Gustafson

\begin{tabular}{|c|c|c|c|c|c|c|}
\hline $\begin{array}{c}\text { Problem size } \\
\text { (MB) }\end{array}$ & \multicolumn{3}{|c|}{$\begin{array}{c}\text { Execution } \\
\text { Time(second) }\end{array}$} & \multicolumn{2}{|c|}{ Speedup } \\
\cline { 1 - 4 } Kosarak & $\mathrm{T}_{1}$ & $\mathrm{~T}_{2}$ & $\mathrm{~T}_{4}$ & $\mathrm{~S}(2)$ & $\mathrm{S}(4)$ \\
\hline 40 & 1178 & 599 & 297 & 1.96 & 3.96 \\
\hline 80 & 2388 & 1208 & 600 & 1.97 & 3.98 \\
\hline 160 & 4860 & 2440 & 1217 & & 1.99 & 3.99 \\
\hline
\end{tabular}

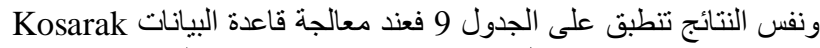

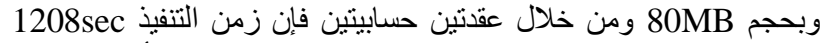

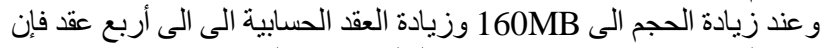

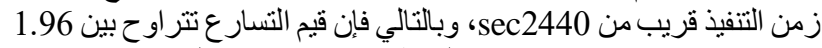

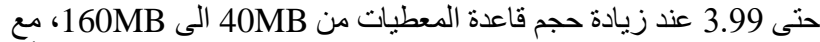

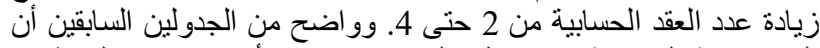

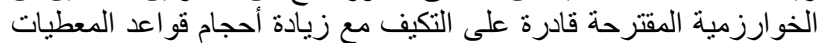
بالاعتماد على زيادة عدد العقد الحسابية في منصة هادية لهادوب والبنية

.MapReduce

المقياس الأخير هو كلفة (cost) الخوارزمية التفر عية والتي تساوي جداء

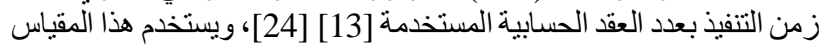
في بر هان كفاءة عملية التفريع عند تحقق المعادل ة6، أي يجب الجاز n معلية على n مقدة حسابية على الأقل.

$$
\mathrm{CP}(\mathrm{n})=n * T_{n} \geq T_{l}
$$

من الجدول 10 نجد أن كلفة الخوارزمية التفر عية المقترحة تحقق المعادل ة6 6

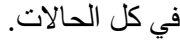

حيث إن T: مثتل زمن التنفيذ التسلسلي للخو ارزمية من خلال عقدة واحدة،

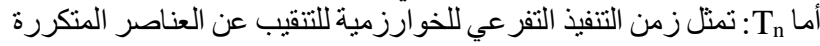

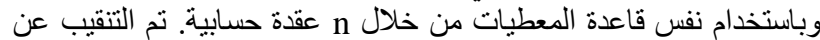

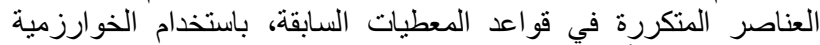

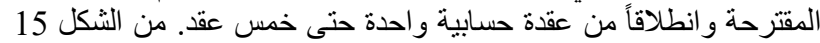

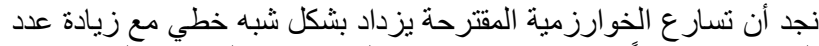

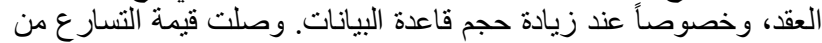

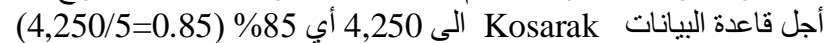
من قيمة التسارع المثالي. بشكل عام من الصعب الثانل الوصول إلى التسارع المثالي نتيجة كلفة الاتصال بين العقد الحسابية] [9] .

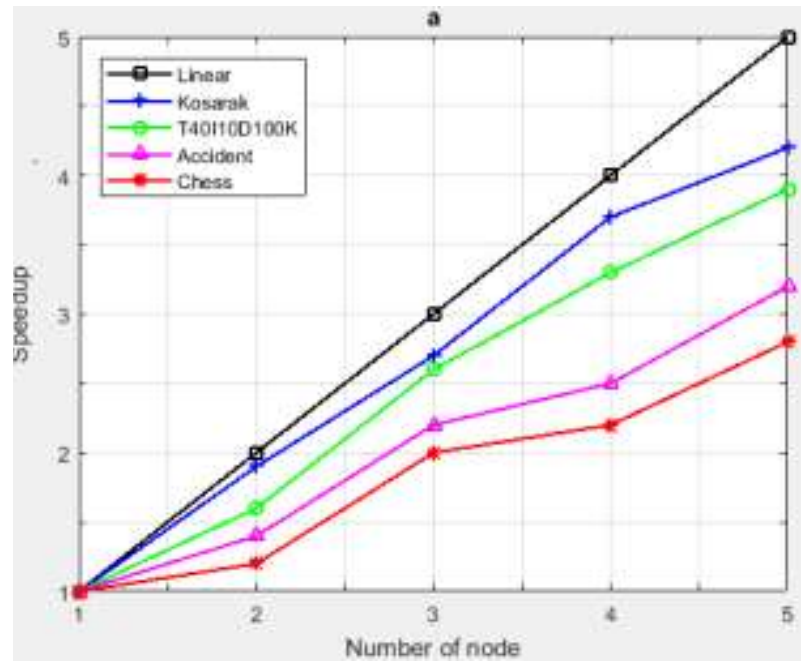

الشكل 15: مقياس Speedup ل للخو ارزمية المقترحة.

المقياس الأخر قابلية التوسع Scaleup [6] [9] وسوف يتم دراسة قابلية

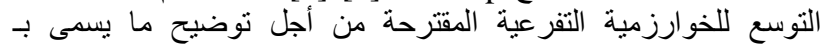
Gustafson's law من خلال قانون Workload Scaling factor

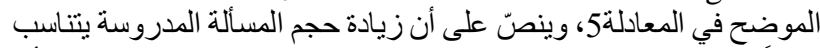

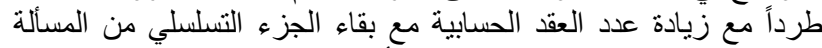

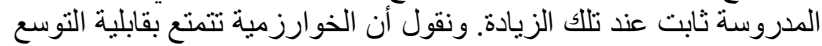
Scalable algorithm)) الحسابية المستخدمة وحجم المسألة المدروسة [13] [24] .

$$
\text { Speedup }=\mathrm{S}(\mathrm{n})=\frac{T 1}{T n}=1+(n-1) * p^{*}
$$

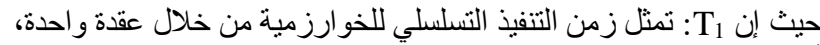

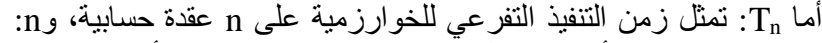

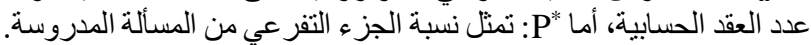

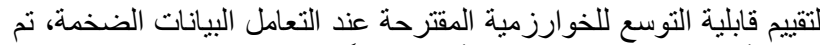

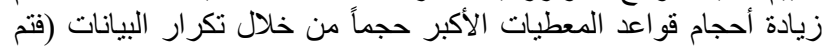

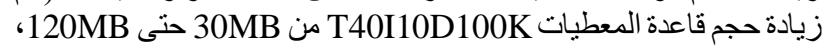
ومن 40MB حتى 160MB لقاعدة المعطيات Kosarak)، وبادة وبالمقابل نم زيادة عدد العقد من 1 إلى 2 ومن ثم 4 عقد، ولم ينت استخدام قاعدة المعطيات

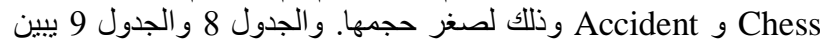
زمن التنفيذ وقيم مقياس التسار ع وفق قانون Gustafson's law. 
[2] Apache Hadoop. http://hadoo p. apach e.org/. Accessed 8 Oct 2019.

[3] Apiletti D., Baralis E., Cerquitelli T., Garza P., Pulvirenti F., and Venturini L., 2017, "Frequent itemsets mining for big data: a comparative analysis," Big Data Research, vol. 9, pp. 67-83.

[4] Alhamodi A. A. G. and Lu S., 2016," MRFP: Discovery Frequent Patterns Using MapReduce Frequent Pattern Growth", IEEE, International Conference on Network and Information Systems for Computers, page 298-301, 978-1-4673-8838-2/16 \$31.00@2016 IEEE, DOI 10.1109/ICNISC.2016.37.

[5] Dahdouh K., Dakkak A., Oughdir L. and Messaoudi F., 2019. 'Large-scale e-learning recommender system based on Spark and Hadoop'. Springer, Journal of big data (2019) 6:2, http://doi.org/10.1186/s40537-019-0169-4.

[6] Gassama A.D.D., Camara F. and Ndiaye S.2017," SFPG: A Parallel Version of FP-growth Algorithm under Apache Spark TM", the second IEEE International Conference on Cloud Computing and Big Data Analysis, 978-1-5090-4499-3/17/\$31.00 (C)2017 IEEE.

[7] Han, J., Pei, J. and Yin .2000 'Mining frequent patterns without candidate generation', in Proceedings of the ACM-SIGMOD Conference Management of Data, Vol. 29, No. 2, pp.1-12.

[8] Han, J., Pei, J., Yin, Y. and Mao, R. 2004 'Mining frequent patterns without candidate generation: $a$ frequent-pattern tree approach', Data Mining and Knowledge Discovery, Vol. 8, No. 1, pp.53-87.

[9] Ji S., Zhang D. and Zhang L, 2016," Paths sharing based FP-growth data mining algorithms", IEEE, 978-1-5090-2860-3/16/\$31.00 @2016 IEEE.

[10] Jiang H. and Meng H., 2017," A Parallel FPgrowth Algorithm Based on GPU", the Fourteenth IEEE International Conference on e-Business Engineering, 978-1-5386-1412-9/17 \$31.00@2017IEEE, DOI 10.1109/ICEBE. 2017.24.

[11] Jian L., Wang C., Liu Y., Liang S., Yi W., and Shi Y., 2013, "Parallel data mining techniques on graphics processing unit with compute unified device architecture (cuda)," The Journal of Supercomputing, vol. 64, no. 3, pp. 942-967.

[12] Kulkarni P. G. and Khonde S. R., 2017, "HDFS Framework for Efficient Frequent Itemset Mining Using MapReduce", IEEE, 978-1-50904264-7/17/\$31.00 @2017 IEEE.

[13] Kwiatkowski J. and Olech L. P., 2016, "Scalability Model Based on the Concept of Granularity", arXiv:1604.00554v1[cs. DC], 2 Apr 2016.
الجدول 10: مقياس الكلفة Cost للخوارزمية المقترحة.

\begin{tabular}{|c|c|c|c|}
\hline Dataset & $\mathbf{T}_{\mathbf{1}}(\mathbf{s e c})$ & $\mathbf{T}_{\mathbf{5}}(\mathbf{S e c})$ & $\mathbf{C P ( 5 ) = 5 * \mathbf { T } _ { \mathbf { 5 } }}$ \\
\hline Chess & 162 & 35 & 175 \\
\hline Accident & 197 & 42 & 210 \\
\hline T40I10D100K & 466 & 95 & 475 \\
\hline Kosarak & 1178 & 240 & 1200 \\
\hline
\end{tabular}

$$
\text { الاستنتاجات والتوصيات: }
$$

9

تم في هذا البحث دراسة لخوارزميات التنقيب عن العناصر المتكررة خاصة

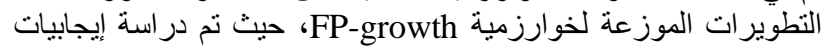

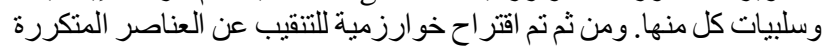

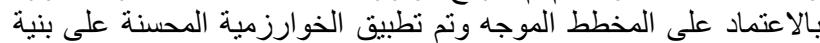

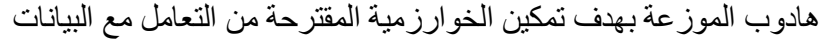
الضخمة، ويمكن تلخيص النتائج كمايلي:

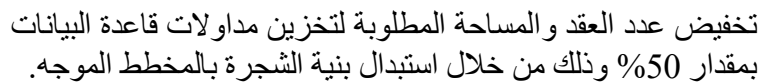

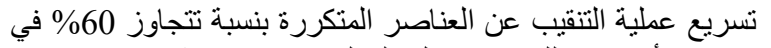

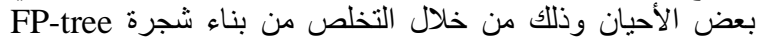

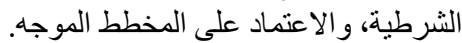

تم تخفيض التعقيد الحسابي والاتصال بين عقد التجمع وارسال

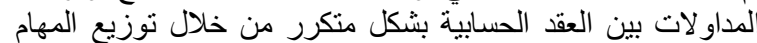

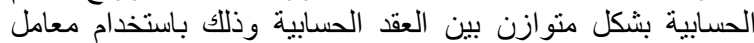

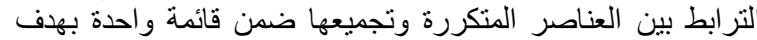
تقسيم المداو لات بين العقد الحسابية.

أكدت النتائج التجربية و التي طُبقت على قو اعد معطيات مختلفة سواء

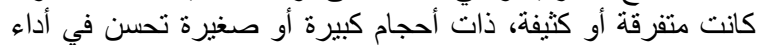

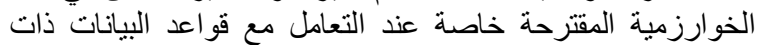

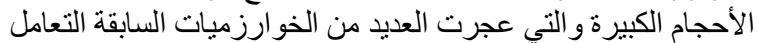
مeهt.

5. ت تتمتع الخوارزمية المقترحة بتسارع شبه خطي في معظم الحالات

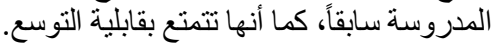

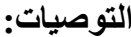

على الرغم من فعالية الخوارزمية المقترحة، الا أنه لاز ال هناك العديد من التحسينات المستقبلية أهمها:

1. مكين الخوارزمية المقترحة من التعامل مع تيار بيانات مستمرة

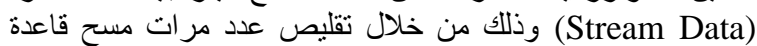

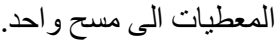

تسريع عملية تجميع المداولات المتشابهة وذلك من خلال استخدام تقنيات LSH-based Partitioning. .2

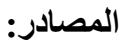

[1] Agrawal, R., \& Srikant, R. 1994. "Fast algorithm for mining association rules in large databases". In Proceedings of 20th VLDB conference (pp. 487499). 
mining association rules". IEEE Transactions on Knowledge and Data Engineering, 9(5), 813-825.

[20] Shirke D., Varshney D. 2016," A Survey on Techniques of Parallel Mining of Frequent Itemsets Using MapReduce", International Journal of Advance Research in Computer Science and Management Studies, ISSN: 2321 -7782 (Online).

[21] Tanbeer S.K., Chowdhury F.A., Jeong B. and Lee, Y-K. 2008 "Efficient single-pass frequent pattern mining using a prefix-tree", in Information Sciences, Vol. 179, No. 5, pp.559-583.

[22] Tehreem A., Khawaja S.G., Akram M.U., Khan S. A. and Ali M., 2017," Parallel Architecture for Implementation of Frequent Itemset Mining Using FP-growth", IEEE, 2017 International Conference on Signals and Systems (ICSigSys), 9781-5090-6748-0/17/\$31.00 @2017 IEEE.

[23] Xun Y., Zhang J., Qin X. and Zhao X.,2016,"FiDoop-DP: Data Partitioning in Frequent Itemset Mining on Hadoop Clusters", IEEE, 1045-9219 (c) 2016 IEEE.

[24] Woodside M. and Jogalekar P., 2000," Evaluating the Scalability of Distributed Systems", IEEE Transactions on Parallel and Distributed Systems, vol. 11, NO. 6, June2000, pp.589-603.

[25] FIMI 2004, FIMI Repository [online] http://fimi.cs.helsinki.fi/data Accessed 8 Oct 2019.
[14]Li H., Wang Y., Zhang D., Zhang M., and Chang E. Y., 2008," PFP: Parallel FP-Growth for Query Recommendation". In Proceedings of the 2008 ACM conference on Recommender systems.

[15] Makanju A., Farzanyar Z., An A., Cercone N., Hu Z. Z. and $\mathrm{Hu}$ Y., 2016," Deep Parallelization of Parallel FP-growth Using Parent-Child MapReduce", IEEE, International Conference on Big Data, pp. 1422- 1431.

[16] Ma Z., Yang J., Zhang T. and Liu F. 2016," An Improved Eclat Algorithm for Mining Association Rules Based on Increased Search Strategy", International Journal of Database Theory and Application, pp.251-266, Vol.9, ISSN: 2005-4270 IJDTA, No.5.

[17] Martire I., Da Silva P. N., Plastino A. and Fabris F., 2017,"A novel probabilistic Jaccard distance measure for classification of sparse and uncertain data", Symposium on Knowledge Discovery, Mining and Learning, KDMILE 2017.

[18] Nikam P. V., Deshpande D. S., 2018," New approach in Big Data Mining for frequent itemset using mapreduce in HDFS", IEEE, 3rd International Conference for Convergence in Technology (I2CT), 978-1-5386-4273-3 /18/ \$31.00 (C)2018 IEEE.

[19] Park, J. S., Chen, M. S., \& Yu, P. S. 1997. "Using a hash-based method with transaction trimming for 


\title{
Frequent Itemset Mining Based on Development of FP-growth Algorithm and Use MapReduce Technique
}

\author{
Zakria Mahrousa ${ }^{1}$, Dima Mufti Alchawafa ${ }^{2}$, and Hasan Kazzaz ${ }^{3 *}$ \\ ${ }^{1}$ Department of Computer Engineering, Faculty of Electrical and Electronic Engineering, University of Aleppo, Syria, Rmahrousa@hotmail.com. \\ ${ }^{2}$ Department of Computer Engineering, Faculty of Electrical and Electronic Engineering, University of Aleppo, Syria, dima.mufti@gmail.com. \\ 3 Department of Computer Engineering, Faculty of Electrical and Electronic Engineering, University of Aleppo, Syria, kazzazhasan @ gmail.com. \\ *Corresponding author: Hasan Kazzaz, kazzazhasan@gmail.com. \\ Published online: 31 March 2021
}

Abstract- The Finding of frequent itemset in big data is an important task in data mining and knowledge discovery. The exponential daily growth of data, called "Big Data", mining frequent patterns from the huge volumes of data has many challenges due to memory requirement, multiple data dimensions, heterogeneity of data and so on. The complexities related to mining frequent item-sets from a Big Data can be minimized by using Modified FP-growth algorithm and parallelizing the mining task with Map Reduce framework in Hadoop. In this paper, a modified FP-growth based on directed graph with Hadoop framework will reduce the execution time for the massive database and works efficiently on number of nodes (computers). The algorithm was tested, our experimental results demonstrated that the proposed algorithm could scale well and efficiently process large datasets. In addition, it achieves improvement in memory consumption to store frequent patterns and time complexity.

Keywords- Association Rule Mining (ARM), Big Data, Map-Reduce, FP-growth, Directed Graph. 